Linköping Studies in Science and Technology.

Dissertation No. 1846

\title{
Admissible transformations and the group classification of Schrödinger equations
}

\author{
Célestin Kurujyibwami
}

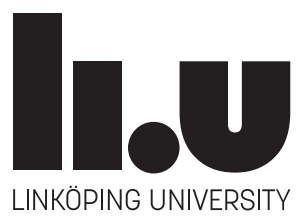

Department of Mathematics,

Division of Mathematics and Applied Mathematics

Linköping University, SE-581 83 Linköping, Sweden

Linköping 2017 
Linköping Studies in Science and Technology.

Dissertation No. 1846

\section{Admissible transformations and the group classification of Schrödinger equations}

Copyright @ C Célestin Kurujyibwami, 2017

Division of Mathematics and Applied Mathematics

Department of Mathematics

Linköping University

SE-581 83 Linköping, Sweden

celestin.kurujyibwami@liu.se

ISSN 0345-7524ＩSBN 978-91-7685-540-9

Printed by LiU-Tryck, Linköping, Sweden 2017 


\section{Dedication}

To my family, Dancille Mukarugina,

Hope Benigne Ineza, Dalton Bruce Hirwa, For your patience during my studies. 



\section{Abstract}

We study admissible transformations and solve group classification problems for various classes of linear and nonlinear Schrödinger equations with an arbitrary number $n$ of space variables.

The aim of the thesis is twofold. The first is the construction of the new theory of uniform semi-normalized classes of differential equations and its application to solving group classification problems for these classes. Point transformations connecting two equations (source and target) from the class under study may have special properties of semi-normalization. This makes the group classification of that class using the algebraic method more involved. To extend this method we introduce the new notion of uniformly semi-normalized classes. Various types of uniform semi-normalization are studied: with respect to the corresponding equivalence group, with respect to a proper subgroup of the equivalence group as well as the corresponding types of weak uniform semi-normalization. An important kind of uniform semi-normalization is given by classes of homogeneous linear differential equations, which we call uniform semi-normalization with respect to linear superposition of solutions.

The class of linear Schrödinger equations with complex potentials is of this type and its group classification can be effectively carried out within the framework of the uniform semi-normalization. Computing the equivalence groupoid and the equivalence group of this class, we show that it is uniformly semi-normalized with respect to linear superposition of solutions. This allow us to apply the version of the algebraic method for uniformly semi-normalized classes and to reduce the group classification of this class to the classification of appropriate subalgebras of its equivalence algebra. To single out the classification cases, integers that are invariant under equivalence transformations are introduced. The complete group classification of linear Schrödinger equations is carried out for the cases $n=1$ and $n=2$.

The second aim is to study group classification problem for classes of generalized nonlinear Schrödinger equations which are not uniformly semi-normalized. We find their equivalence groupoids and their equivalence groups and then conclude whether these classes are normalized or not. The most appealing classes are the class of nonlinear Schrödinger equations with potentials and modular nonlinearities and the class of generalized Schrödinger equations with complex-valued and, in general, coefficients of Laplacian term. Both these classes are not normalized. The first is partitioned into an infinite number of disjoint normalized subclasses of three kinds: logarithmic nonlinearity, power nonlinearity and general modular nonlinearity. The properties of the Lie invariance algebras of equations from each subclass are studied for arbitrary space dimension $n$, and the complete group classification is carried out for each subclass in dimension $(1+2)$. The second class is successively reduced into subclasses until we reach the subclass of $(1+1)$ dimensional linear Schrödinger equations with variable mass, which also turns out to be non-normalized. We prove that this class is mapped by a family of point transformations to the class of $(1+1)$-dimensional linear Schrödinger equations with unique constant mass. 



\section{Populärvetenskaplig sammanfattning}

I denna avhandling studerar vi hur man kan klassificera både linjära och icke-linjära Schrödinger ekvationer med gruppsymmetri som klassificeringsprincip. Detta leder till introduktionen av en ny teori av så kallade uniformt halv-normaliserade klasser av differentialekvationer som kan tillämpas på de olika typer av ekvationsklasser. I synnerhet tillämpas metoden på linjära Schrödinger ekvationer med potential (både reell och komplex) och på så vis erhållas en fullständig klassificering av denna typ av Schrödinger ekvationer i dimension $1+1$ och $1+2$.

Även icke-linjära Schrödinger ekvationer, med vissa typer av icke-linjära termer, studeras och en fullständig klassificering erhållas för dessa icke-linjära termer i dimension 1+2. De klasser av icke-linjära Schrödinger ekvationer som studeras här innehåller en ekvationsklass med variabel massa. Vi visar bland annat att i dimension 1+1 varje Schrödinger ekvation med variabel massa kan avbildas på en Schrödinger ekvation med konstant massa.

Både linjära och icke-linjära Schrödinger ekvationer förekommer i fysik och teknik, och även linjära Schrödinger ekvationer med komplex potential har på senare år undersökts i kvantteori. Att klassificera sådana ekvationer enligt gruppsymmetriska egenskaper är att skapa ett slags ordning i en skenbar oordning, och det visar sig att det i grunden finns bara ett ändligt antal "grundtyper" av Schrödinger ekvationer som är sinsemellan inekvivalenta. 



\section{Acknowledgments}

First of all I would like to express my deepest gratitude to my supervisors, Professor Peter Basarab-Horwath and Professor Roman Popovych for their invaluable impact on my research in Mathematics. They introduced me to the subject and facilitated my research by providing me the required support along the way. I am very grateful for the academic environment we have worked with and all valuable advice and comments for the present thesis.

I thank Bengt-Ove Turesson, Björn Textorius, Meaza, Theresa and all the academic and administrative staffs of the Department of Mathematics for their help and guidance whenever needed.

I express my gratitude to the International Science Programme (ISP) for their financial support through the East African Universities Mathematics Programme (EAUMP)-network. In particular, I would like to thank Leif Abrahamsson for his advice and care concerning the welfare students supported by the above programme. Further my thanks go to Pravina Gajjar, Dr Karangwa Desiré, Michael Gahirima and Dr Banzi Wellars for their quick responses and advice whenever needed.

Prof Alexandre Lyambabaje, Dr Mahara Isidore, Dr Minani Froduard and Mr Claudio Achola showed me interest in doing Mathematics from the very beginning in my graduate and post-graduate studies. Thank you very much.

My thanks go to my fellow PhD students for making Linköping such a nice place to live and work. In particular, I express my thanks to Adson Banda, Venuste Nyagahakwa, Innocent Ngaruye and Béatrice Byukusenge, for their discussion during my $\mathrm{PhD}$ studies.

I want also to thank the Government of Rwanda and the University of Rwanda for giving me the opportunity to pursue further studies and providing me study leave.

Finally, I am grateful to my family and friends for their strong support. Thank you mam for your love and faith in me. Thank you my wife Dancille and my children Ineza and Hirwa for your encouragement and your accepting for the pain of missing me during the studies. 



\section{List of papers}

The thesis consists of five papers, which will be referred to in the text by their numbers in Roman numerals.

I. Kurujyibwami C., Basarab-Horwath P. and Popovych R.O., Algebraic method for group classification of $(1+1)$-dimensional linear Schrödinger equations, submitted to Acta Appl. Math., (2016) arXiv:1607.04118, 30 pp.

II. Kurujyibwami C., Basarab-Horwath P. and Popovych R.O., Group classification of multidimensional linear Schrödinger equations with the algebraic method, (in preparation) (2017).

III. Kurujyibwami C., Basarab-Horwath P. and Popovych R.O., Group classification of multidimensional nonlinear Schrödinger equations, (in preparation) (2017).

IV. Kurujyibwami C., Basarab-Horwath P. and Popovych R.O., Admissible transformations of $(1+1)$-dimensional Schrödinger equations with variable mass, (in preparation) (2017).

V. Kurujyibwami C., Equivalence groupoid for $(1+2)$-dimensional linear Schrödinger equations with complex potentials, Journal of Physics: Conference Series, 621 (2015),12008-12014.

\section{Contribution}

Authors's contribution to these papers is the following. I wrote Paper V myself. In the other four papers supervisors posed the problems to be studied and proposed the general plan of their solutions. The other piece of the work, which includes computing and formulating results and writing the papers, was done by me.

The results of the above papers were presented by me at four conferences:

- Group classification of multidimensional Schrödinger equations with potentials and general modular nonlinearity, The 3rd EAUMP conference "Advances in Mathematics and its applications" (October 26-28, 2016, Makerere University, Kampala, Uganda).

- Group classification of multidimensional nonlinear Schrödinger equations, Eighth International Workshop "Group Analysis of Differential Equations and Integrable Systems" (June 12-17, 2016, Larnaka, Cyprus).

- Group classification of multidimensional linear Schrödinger equations with algebraic method, 14th conference "Mathematics in Technical and Natural Sciences" (September 18-24, 2015, Koscielsco, Poland).

- Algebraic method for group classification of $(1+1)$-dimensional linear Schrödinger equations, Seventh International Workshop "Group Analysis of Differential Equations and Integrable Systems" (June 15-19, 2014, Larnaka, Cyprus). 
These results were also presented in various seminars:

- Group classification of linear Schrödinger equations by the algebraic method, Licentiate seminar at the Department of Mathematics (February 24, 2016, Linköping University, Linköping, Sweden).

- Group classification of (1+n)-multidimensional linear Schrödinger equations, Seminar at the Department of Mathematics (January 21, 2016, University of Rwanda, Kigali, Rwanda).

- Group classification of (1+1)-dimensional linear Schrödinger equations, Mathematics colloquium (May 23, 2014, Linköping University, Linkoping, Sweden).

- Group classification of Schrödinger equations,

First Network Meeting for SIDA and ISP funded PhD Students in Mathematics (March 7-8, 2017, Stockholm, Sweden). 


\section{Contents}

1 Introduction 3

1.1 Lie symmetries of Schrödinger equations: known results . . . . . . 5

1.2 Motivation and relevance $\ldots \ldots \ldots \ldots \ldots$

2 Basic notions of symmetry analysis $\quad 9$

2.1 One-parameter groups and their infinitesimal generators. . . . . . 9

2.2 Jet bundles . . . . . . . . . . . . . . . . . . . 10

2.3 Lie symmetries of differential equations . . . . . . . . . . . . . 13

2.3 .1 Nondegenerate systems of differential equations . . . . . . . 16

3 Classes of differential equations and equivalence transformations 17

3.1 Classes of differential equations . . . . . . . . . . . . . . . 17

3.2 Admissible transformations . . . . . . . . . . . . . . . . . . . . 19

3.3 Equivalence groups . . . . . . . . . . . . . . . . . . . . 20

3.3 .1 Usual equivalence group and equivalence algebra . . . . . . 20

3.3.2 Generalized and generalized extended equivalence groups . 21

3.3.3 Gauge equivalence group . . . . . . . . . . . . . . . 23

3.3.4 Conditional equivalence groups . . . . . . . . . . . . . . . . 24

3.4 Normalization properties . . . . . . . . . . . . . . . . . . 24

\begin{tabular}{|lll}
4 & Group classification problem & 27
\end{tabular}

4.1 Formulation of the problem . . . . . . . . . . . . . . . 27

4.2 Methods to solve the problem of group classification . . . . . . . . 27

4.2.1 Infinitesimal Lie's method and direct integration of

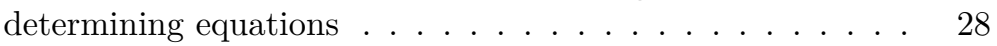

$4.2 .2 \quad$ Algebraic method for group classification . . . . . . . . . . 28 
4.3 Algorithm for solving the problem of group classification . . . . . . 30

$\begin{array}{|ll|}5 & \text { Summary of the papers }\end{array}$

\begin{tabular}{ll}
\hline Bibliography & 35
\end{tabular}

\begin{tabular}{|ll|l|}
\hline I Algebraic method for group classification of & \\
\hline$(1+1)$-dimensional linear Schrödinger equations & 41 \\
\hline
\end{tabular}

$1 \quad$ Introduction $\ldots \ldots \ldots \ldots 42$

2 Group classification in classes of differential equations . . . . . . . 45

3 Uniformly semi-normalized classes . . . . . . . . . . . . . . . . 48

$4 \quad$ Equivalence groupoid. . . . . . . . . . . . . . . . 53

$5 \quad$ Analysis of determining equations for Lie symmetries . . . . . . . . 58

$6 \quad$ Group classification. . . . . . . . . . . . . . . . 61

$7 \quad$ Alternative proof . . . . . . . . . . . . . . . . . . 68

8 Subclass with real-valued potentials . . . . . . . . . . . . . . . . . . 72

9 Conclusion ............................ 75

\begin{tabular}{lr}
\hline References & 77
\end{tabular}

\begin{tabular}{|lr|}
\hline II Group classification of multidimensional linear Schrödinger \\
\hline equations with the algebraic method & 81
\end{tabular}

1 Introduction . . . . . . . . . . . . . . . . 82

2 Basics notions of group classification . . . . . . . . . . . . . . . . 84

3 Uniformly semi-normalized classes . . . . . . . . . . . . . . . . . . 86

$4 \quad$ Weakly uniformly semi-normalized classes . . . . . . . . . . . . . . 91

$5 \quad$ Equivalence groupoid . . . . . . . . . . . . . . . . . . . . 93

$6 \quad$ Equivalence group and equivalence algebra. . . . . . . . . . . . . . 96

$7 \quad$ Analysis of determining equations for Lie symmetries . . . . . . . . 98

$8 \quad$ Further properties of Lie symmetry algebras . . . . . . . . . . . . . 101

$9 \quad$ Group classification of $(1+2)$-dimensional linear Schrödinger

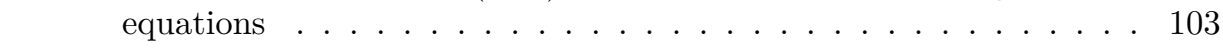

10 Conclusion . . . . . . . . . . . . . . . . . . . . 113

$\begin{array}{ll}\text { References } & 115\end{array}$

\begin{tabular}{|cc|}
\hline III Group classification of multidimensional nonlinear & 119 \\
\hline Schrödinger equations & 119
\end{tabular}

1 Introduction . . . . . . . . . . . . . . . . . . . . 120

$2 \quad$ Equivalence groupoid . . . . . . . . . . . . . . . . . . . . . 122

$3 \quad$ Equivalence group and equivalence algebra. . . . . . . . . . . . . . 124

$4 \quad$ Determining equations for Lie symmetries . . . . . . . . . . . . 128

$5 \quad$ Schrödinger equations with potentials and modular nonlinearity. . 131

5.1 General case ................... 131

$5.2 \quad$ Logarithmic modular nonlinearity . . . . . . . . . . . . 132

$5.3 \quad$ Power nonlinearity . . . . . . . . . . . . . . . 133

6 Group classification in dimension $(1+2) \ldots$. . . . . . . . . . 135 
$6.1 \quad$ General case of nonlinearity . . . . . . . . . . . . . . 136

6.2 Logarithmic modular nonlinearity . . . . . . . . . . . . . . 140

6.3 Power nonlinearity . . . . . . . . . . . . . . . . 145

7 Conclusion . . . . . . . . . . . . . . . . . . . . . 149

\begin{tabular}{ll}
\hline References & 151
\end{tabular}

IV Admissible transformations of (1+1)-dimensional Schrödinger equations with variable mass $\quad \mathbf{1 5 5}$

1 Introduction . . . . . . . . . . . . . . . . . 156

$2 \quad$ Equivalence groupoids of covering classes . . . . . . . . . . . . . . 159

3 Admissible transformations in narrower classes . . . . . . . . . . . 163

$4 \quad$ Linear Schrödinger equations with variable mass . . . . . . . . . . 166

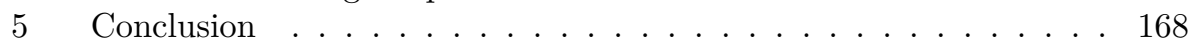

\begin{tabular}{lr}
\hline References & 169
\end{tabular}

\begin{tabular}{|ll|}
\hline V & Equivalence groupoid for $(1+2)$-dimensional linear \\
\hline Schrödinger equations with complex potentials & 171 \\
\hline
\end{tabular}

$1 \quad$ Introduction . . . . . . . . . . . . . . . . . . . . . . . . . . . . 172

$2 \quad$ Equivalence groupoid . . . . . . . . . . . . . . . . . . . . . . . . . . . . 174

$3 \quad$ Equivalence group and normalization properties . . . . . . . . . . . 177

4 Conclusion . . . . . . . . . . . . . . . . . 177

\begin{tabular}{lr}
\hline References & 179
\end{tabular} 

Theoretical background 



\section{1}

\section{Introduction}

Lie symmetries of differential equations were first introduced by the Norwegian mathematician Sophus Lie in his efforts to create a theory for differential equations similar to the work of Galois on algebraic equations. In this approach, a symmetry of a differential equation (ordinary or partial) is an invertible transformation that maps the set of solutions of the equation to itself: if $L(u)=0$ is a differential equation, where $u$ stands for a solution of the equation, then we must have

$$
L(\tilde{u})=0 \quad \text { whenever } \quad L(u)=0,
$$

where $\tilde{u}$ is the transform of $u$. It is easy to see that the set of all symmetries of a given equation form a group.

Lie's theory gave birth to the theory of continuous transformations group and then led to the concept of Lie groups: a Lie group is, in modern terminology, a finite-dimensional analytic manifold whose underlying set is also a group so that the group operations of multiplication and taking the inverse, $(g, h) \rightarrow g h$, $g \rightarrow g^{-1}$, are analytic (see [45]).

Lie's original work on symmetries of differential equations was done in terms of what are now called Lie algebras and one-parameter groups. These one-parameter groups are generated by vector fields (known also as infinitesimal symmetries) and the vector fields form a Lie algebra. The first step in a (Lie) symmetry analysis of a given differential equation is to find the vector fields that generate (local) one-parameter groups of symmetry transformations. To do this, Lie developed an algorithmic method of determining these vector fields. This was one of his many deep results, and it is still the basis of every investigation of the symmetry properties of differential equations. This is known as the Lie infinitesimal method. Having found the Lie algebra of infinitesimal symmetries of an equation, one can then reconstruct the local one-parameter groups that they generate, and from a 
given solution of the equation we can generate new ones by applying the local symmetry transformations.

In his approach, Lie posed two questions:

1. What symmetries does a given equation possess?

2. Given a group (in a given representation), what differential equations (of a given order and type) admit this group as a group of symmetries?

In this thesis, we look at a variant of the first question: given a class of differential equations, what symmetries can the equations in that class possess and how can we classify them according to the various symmetry properties that they have?

From 1899, the year in which Lie died, up to the 1950's, Lie's methods fell almost into disuse. However, two notable exceptions were Bateman's proof in 1909 that the free wave equation is invariant under the conformal group and Emmy Noether's work on the correspondence between the symmetries of a Lagrangian variational problem and the conservation laws of the Euler-Lagrange equations 32 .

In the late 1950's and early 1960's, Ovsiannikov began publishing his work on the symmetry analysis of differential equations ([34, 35]) and from the late 1960 's to the present day there has been an exponential growth in the application of symmetry methods to differential equations (see for instance [3], [6], 33], 36], 37] and the references cited there).

Although Lie's symmetry algorithm based on the infinitesimal Lie method is fundamental in symmetry analysis, it is not always powerful enough on its own to give complete results. For classes of differential equations whose arbitrary elements depend on one variable only but not on any derivative, such as the equation

$$
u_{t}=f(u) u_{x x}+f_{u}(u) u_{x}
$$

this approach is successful. However, the method is not powerful enough when confronted by equations of the type

$$
u_{t}=f\left(u, u_{x}\right) u_{x x}+g\left(u, u_{x}\right)
$$

The reason for this is quite simple: the infinitesimal method of Lie depends on being able to express the symmetry condition (on the coefficients of a symmetry vector field) as a polynomial in the derivatives. This is not possible when terms such as $f\left(u, u_{x}\right)$ and $g\left(u, u_{x}\right)$ are present. The way out of this problem is to supplement Lie's infinitesimal method with other techniques. In this thesis we use the algebraic method [4, which is based on the subgroup analysis of the equivalence group associated with a class of differential equations under study. This approach uses a synthesis of Lie's infinitesimal method, the technique of equivalence transformations [37] and the analysis of low-dimensional subalgebras as well as the equivalence groupoid of the equation (or class of equations) [0]. 


\subsection{Lie symmetries of Schrödinger equations: known results}

The study of Schrödinger equations from the symmetry point of view began in the early 1970's with the symmetry analysis of the linear Schrödinger equation ([7], 25], 27], 28, 29], 30]). In [27] Niederer found the usual Lie symmetry group of (1+3)-dimensional free linear Schrödinger equations using Lie's infinitesimal method. In subsequent papers $([28$, [29, 30] $)$ he obtained the maximal kinematical invariance group of the harmonic oscillator as well establishing an isomorphism between the invariance groups for both the harmonic and linear potential, and the free invariance particle. Niederer also obtained a symmetry classification of linear Schrödinger equations with arbitrary real potential $V=V(t, x)$ for space-dimension less or equal to three. Boyer ([7]) solved the problem of group classification of the class of linear Schrödinger equations with arbitrary real-valued time independent potential and found a generalization of Niederer's results. In the book [25], Miller gave a résumé of his important work on the symmetry properties and separation of variables for linear Schrödinger equations.

In the early 1990's, Fushchych and his collaborators in ([13], [14, [15], [16]) studied the symmetries and conditional symmetries and exact solutions of Schrödinger equations of the form

$$
i \psi_{t}+\lambda \Delta \psi+F(|\psi|) \psi=0 \quad \lambda \neq 0
$$

(as well as considering other nonlinear equations). Earlier, in ([17, [18, [19] the group invariant solutions of $(1+3)$-dimensional generalized quintic nonlinear Schrödinger equations, with $F=a_{0}+a_{1}|\psi|^{2}+a_{2}|\psi|^{4}$ were constructed. The class $\mathcal{F}$ of nonlinear Schrödinger equations of the type

$$
i \psi_{t}+\psi_{x x}+F\left(t, x, \psi, \psi^{*}, \psi_{x}, \psi_{x}^{*}\right)=0,
$$

where $F$ is an arbitrary smooth, complex-valued function, was studied in ([8]), in particular their invariance under subalgebras of the symmetry algebra of $(1+1)$ dimensional free Schrödinger equation. The symmetry classification for the general case of $F=F\left(\psi, \psi^{*}\right)$ was carried out in [31].

A complete classification of the above type of nonlinear Schrödinger equation with modular nonlinearities, supplementing Lie's infinitesimal approach with the notions of equivalence group and normalized classes of differential equations, was given in [40]. They also gave a complete description of all admissible transformations in the class $\mathcal{F}$ (that is, those that map equations of the class to equations in the same class).

In ([20]) the symmetry analysis of the class of $(1+1)$-dimensional cubic Schrödinger equations with variable coefficients of the form

$$
i \psi_{t}+f(t, x) \psi_{x x}+g(t, x)|\psi|^{2} \psi+h(t, x) \psi=0,
$$

where $f, g, h$ are complex-valued functions of $(t, x)$ with $(\operatorname{Re} f)(\operatorname{Re} g) \neq 0$, was carried out. 
Various other classes of nonlinear Schrödinger equations have over the years been studied using Lie's method supplemented with other techniques. Among these supplementary techniques is the exploitation of equivalence transformations, as developed systematically by Ovsiannikov (see [37). This method has been enhanced and refined by Popovych and his collaborators (for details see [39], 40]). In this thesis, we investigate linear and non-linear Schrödinger equations using the techniques developed by Popovych and his collaborators, and we enhance these methods with new results.

\subsection{Motivation and relevance}

We consider linear and nonlinear Schrödinger equations with complex potentials. Nonlinear Schrödinger equations have been used in nonlinear optics and plasma physics, as well as other fields. Real potentials have an obvious interpretation in quantum mechanics, but recently interest in complex-valued potentials has grown. These complex potentials still give a real-valued spectrum for the Hamiltonian (see for instance [1], [2], [12, [26], 42], 443]).

We are motivated in our treatment of linear and nonlinear Schrödinger equations for a variety of reasons.

First of all, we treat, in the first paper of this thesis, linear Schrödinger equations in (1+1)-time-space with a potential. Although this case was considered previously by Niederer $([27])$ and Boyer $([7])$, their results are not complete. Also, their approach to symmetries was based on an assumption of a linear representation of symmetries (as suggested by representation theory). Here, we use the Lie algorithm for finding the symmetries, and the form of the representation is then dictated by the equation. Our approach gives an exhaustive list of potentials, both real and complex, and we thus obtain completeness in our classification. As mentioned above, some complex potentials have been of interest in various applications, there are complex potentials which still allow the quantum mechanical Hamiltonian to have a real spectrum. In the second paper, we look at the case of linear Schrödinger equations with potential in $(1+n)$-time-space dimensions and then give a more detailed analysis for the $(1+2)$-time-space.

As well as completeness, using our method on this type of Schrödinger equation provides us with a laboratory for the method and the auxiliary concepts we develop. Indeed, we extend the methods described in ([4]). Further, in the third paper we extend the results of $([40])$ to the case of several dimensions.

Since nonlinear Schrödinger equations have been used in many contexts, we use our methods to study nonlinear Schrödinger equations of the form

$$
i \psi_{t}+G\left(t, x, \psi, \psi^{*}, \nabla \psi, \nabla \psi^{*}\right) \Delta \psi+F\left(t, x, \psi, \psi^{*}, \nabla \psi, \nabla \psi^{*}\right)=0, \quad G \neq 0,
$$

and then consider more specific cases of the nonlinearities $F$ and $G$. This study is intended to help in the understanding of the various subclasses of nonlinear equations.

Finally, our study is intended to give a Lie symmetry classification of these Schrödinger equations and to use these Lie symmetries to construct exact solutions of the equations. 
This thesis consists of two parts: in the first part we present a summary of the theoretical background needed to read the research papers and the second part consists of the research papers. 



\section{Basic notions of symmetry analysis}

\subsection{One-parameter groups and their infinitesimal generators.}

Symmetry analysis of differential equations is based on the notion of Lie algebras of vector fields. These vector fields are the generators of (local) Lie groups of symmetry transformations. A symmetry transformation is, as noted in the introduction, simply a transformation which maps a solution of a given differential equation to another solution of the same equation. We begin by recalling some basic concepts and definitions which form the background of our approach. For simplicity, we work in $\mathbb{R}^{n}$ or open subsets of $\mathbb{R}$ as all our considerations in this thesis are of a local nature.

One of the fundamental ingredients in our approach is the concept of (local) one-parameter group. This is just a modification of the idea of a one-parameter group of transformations :

Definition 2.1. 1) A one-parameter group of smooth transformations of $\mathbb{R}^{n}$ (for some positive integer $n$ ) is a one-parameter family of smooth transformations $\Phi_{t}: \mathbb{R}^{n} \rightarrow \mathbb{R}^{n}$ with $t \in \mathbb{R}$, such that $(t, x) \rightarrow \Phi_{t}(x)$ is a smooth map $\mathbb{R} \times \mathbb{R}^{n} \rightarrow \mathbb{R}^{n}$ with the properties that $\Phi_{s} \circ \Phi_{t}=\Phi_{s+t}$ for all $s, t \in \mathbb{R}$ and $\Phi_{0}=\operatorname{id}_{\mathbb{R}^{n}}$.

2) A one-parameter local group of smooth transformations of an open subset $U \subset \mathbb{R}^{n}$ is a family of smooth transformations $\Phi_{t}: U \rightarrow U$ with $t \in I$ for some symmetric interval $I \subset \mathbb{R}$ about $0 \in \mathbb{R}^{n}$ such that $(t, x) \rightarrow \Phi_{t}(x)$ is smooth map $\mathbb{R} \times U \rightarrow U$ and such that $\Phi_{t}(x) \in U$ for all $t \in I$ and $x \in U, \Phi_{0}(x)=x$ for each $x \in U$ and $\Phi_{s} \circ \Phi_{t}(x) \in U$ for $x \in U$ and for all $s, t \in I$ such that $s+t \in I$. 


\section{Example 2.1}

As examples of one-parameter groups of transformations we have

$$
\left[\begin{array}{ll}
\cosh t & \sinh t \\
\sinh t & \cosh t
\end{array}\right], \quad\left[\begin{array}{cc}
\cos t & -\sin t \\
\sin t & \cos t
\end{array}\right], \quad t \in \mathbb{R} .
$$

Clearly, every one-parameter group of transformations is a local one-parameter group. The generator of a local one-parameter group is a vector field which we define as follows:

Definition 2.2. Suppose we are given a local one-parameter group of transformations $\Phi_{t}, t \in I$ on an open subset $U \subset \mathbb{R}^{n}$ as defined in Definition 2.1. Then a vector field $X$ defined on $U$ is the generator of the local one-parameter group on $U$ if $X_{x}$ is the tangent vector to the curve defined by $t \rightarrow \Phi_{t}(x)$ at $t=0$. That is,

$$
X_{x}=\left.\frac{d}{d t} \Phi_{t}(x)\right|_{t=0}
$$

The generator of a local one-parameter group acts on (smooth) functions $f: U \rightarrow \mathbb{R}$ according to the rule

$$
(X f)(x)=\left.\frac{d}{d t} f\left(\Phi_{t}(x)\right)\right|_{t=0}
$$

and this defines a derivation on the multiplicative ring of functions: we have $X(f+g)=X f+X g$ and $X(f g)=(X f) g+f(X g)$, as is easily verified from the definition of the generator $X$.

\subsection{Jet bundles}

Jet bundles, or jet spaces, are the natural setting for discussing differential equations. A jet bundle over a manifold $M$ is essentially a space whose local coordinates are the local coordinates $x=\left(x_{1}, \ldots, x_{n}\right)$ of $M$ together with functions $u=\left(u^{1}, \ldots, u^{m}\right)$ on $M$ as well as their derivatives. Thus, if the manifold is $\mathbb{R}^{2}$ and we have one function $u: \mathbb{R}^{2} \rightarrow \mathbb{R}$, then the jet bundle of order two, denoted by $J^{2}\left(\mathbb{R}^{2}, \mathbb{R}\right)$, is the space with local coordinates $\left(x, u_{(2)}\right)=$ $\left(x_{1}, x_{2}, u, u_{1}, u_{2}, u_{11}, u_{12}, u_{22}\right)$, where $u_{1}$ and $u_{2}$ denote the first-order derivatives $\partial_{1} u$ and $\partial_{2} u$ with $\partial_{1}$ and $\partial_{2}$ being the partial derivatives with respect to $x_{1}$ and $x_{2}$ respectively, and $u_{11}, u_{12}, u_{22}$ denote the three second order partial derivatives of $u: u_{11}=\partial_{1}^{2} u, u_{12}=\partial_{12}^{2} u, u_{22}=\partial_{2}^{2} u$. It is assumed that all mixed derivatives are equal. A rigorous definition of jet bundles and their properties can be found in [38, [41. Our exposition below is informal and follows that of Olver in 33].

For a smooth real-valued function $u\left(x_{1}, \ldots, x_{n}\right)$ of $n$ independent variables we have the $p$ th order derivatives

$$
u_{\alpha}=\frac{\partial^{|\alpha|} u}{\partial x_{1}^{\alpha_{1}} \ldots \partial x_{n}^{\alpha_{n}}},
$$


where $\alpha$ stands for the $n$-tuple of integers $\alpha=\left(\alpha_{1}, \ldots, \alpha_{n}\right)$, and $\alpha_{i}, i=1, \ldots, n$, are nonnegative integers. The tuple $\alpha$ is referred to as a multi-index and its length, denoted by $|\alpha|$, is defined as $|\alpha|=\alpha_{1}+\cdots+\alpha_{n}$. The corresponding jet space is denoted by $J^{p}\left(\mathbb{R}^{n}, \mathbb{R}\right)$ and has local coordinates $(x, u)=\left(x_{1}, \ldots, x_{n}, u\right)$ together with all the partial derivatives $u_{\alpha}, 1 \leqslant|\alpha| \leqslant p$. Thus, for instance, if the underlying space $\mathbb{R}^{2} \times \mathbb{R}$ has local coordinates $(x, y, u)$, then the jet space $J^{3}\left(\mathbb{R}^{2}, \mathbb{R}\right)$ has local coordinates $\left(x, y, u, u_{x}, u_{y}, u_{x x}, u_{x y}, u_{y y}, u_{x x x}, u_{x x y}, u_{x y y}, u_{y y y}\right)$. We define the "zeroth" jet bundle $J^{0}\left(\mathbb{R}^{n}, \mathbb{R}\right)$ as being the space with local coordinates $(x, u)$, the "zeroth" derivative of $u$ being just the function itself.

If we now take a function $u: \mathbb{R}^{n} \rightarrow \mathbb{R}^{m}$ then the corresponding jet space of order $p$ is denoted by $J^{p}\left(\mathbb{R}^{n}, \mathbb{R}^{m}\right)$ and its local coordinates are

$$
(x, u)=\left(x_{1}, \ldots, x_{n}, u^{1}, \ldots, u^{m}\right)
$$

together with all $u_{\alpha}^{a}$ corresponding to the partial derivatives $\partial^{|\alpha|} u^{a} / \partial x_{1}^{\alpha_{1}} \ldots \partial x_{n}^{\alpha_{n}}$, $1 \leqslant|\alpha| \leqslant p, a=1, \ldots, m$. The "zeroth" jet bundle $J^{0}\left(\mathbb{R}^{n}, \mathbb{R}^{m}\right)$ is then the space with local coordinates $(x, u)$. We refer to the space $\mathbb{R}^{n} \times \mathbb{R}^{m}$ with local coordinates $(x, u)$ as the underlying space. It is the graph space of the mapping $u: \mathbb{R}^{n} \rightarrow \mathbb{R}^{m}$. In the literature on symmetries of differential equations the $x=\left(x_{1}, \ldots, x_{n}\right)$ and $u=\left(u^{1}, \ldots, u^{m}\right)$ are called the independent variables and the dependent variables, respectively.

The jet bundles $J^{p}\left(\mathbb{R}^{n}, \mathbb{R}^{m}\right)$ can be given the structure of a differentiable manifold (see [41]) and they are also fibred manifolds: there is a map (the projection map) $\pi_{p}: J^{p}\left(\mathbb{R}^{n}, \mathbb{R}^{m}\right) \rightarrow \mathbb{R}^{n} \times \mathbb{R}^{m}$ which is surjective (it is a submersion) given by $\pi_{p}\left(x, u_{(p)}\right)=(x, u)$, where $u_{(p)}$ stands for the collection of all partial derivatives of $u$ up to and including order $p$, with the "zeroth" derivatives being just the functions themselves. The fibre above $(x, u)$ is just the inverse image $\pi_{p}^{-1}(x, u)$ (see [38, [41]). One also has the projections $\pi_{p, l}: J^{p}\left(\mathbb{R}^{n}, \mathbb{R}^{m}\right) \rightarrow J^{q}\left(\mathbb{R}^{n}, \mathbb{R}^{m}\right)$ for $p \geqslant q$ given by $\pi_{p, q}\left(x, u_{(p)}\right)=\left(x, u_{(q)}\right)$ and it is easy to verify that $\pi_{j, p} \circ \pi_{p, q}=\pi_{j, q}$ whenever $j \geqslant p \geqslant q$. We also have $\pi_{p, p}=$ id and $\pi_{p}=\pi_{l} \circ \pi_{p, q}$ for all $p \geqslant q$.

The structure of a fibred manifold allows us to define diffeomorphisms of $J^{p}\left(\mathbb{R}^{n}, \mathbb{R}^{m}\right)$. Note that any diffeomorphism $\Phi: \mathbb{R}^{n} \times \mathbb{R}^{m} \rightarrow \mathbb{R}^{n} \times \mathbb{R}^{m}$ of the underlying space $\mathbb{R}^{n} \times \mathbb{R}^{m}$ induces a transformation of the derivatives of all orders of $u=\left(u^{1}, \ldots, u^{m}\right)$. The induced transformation on the jet space $J^{p}\left(\mathbb{R}^{n} \times \mathbb{R}^{m}\right)$ obtained in this way (by the usual chain rule) is called the $p$ th prolongation of $\Phi$. It is clear that the $p$ th prolongation of a diffeomorphism $\Phi$ will depend only on the local coordinates $\left(x, u_{(p)}\right)$. We use the notation $\Phi_{(p)}$ to denote the $p$ th prolongation of $\Phi$.

The jet bundles described above are endowed with differential operators $D_{i}$, $i=1, \ldots, n$ called the total derivatives with respect to $x_{i}$. They are defined as $D_{i}=\partial_{i}+u_{\alpha+\delta_{i}}^{a} \partial_{u_{\alpha}^{a}}$, where $\delta_{i}$ is the multi-index whose $i$ th entry equals 1 and whose other entries are zeroes. Thus, $u_{\alpha+\delta_{i}}^{a}$ with $\alpha=\left(\alpha_{1}, \ldots, \alpha_{n}\right)$ stands for the derivative

$$
\frac{\partial u_{\alpha}^{a}}{\partial x_{i}}=\frac{\partial^{|\alpha|+1} u^{a}}{\partial x_{1}^{\alpha_{1}} \ldots \partial x_{i-1}^{\alpha_{i-1}} \partial x_{i}^{\alpha_{i}+1} \partial x_{i+1}^{\alpha_{i+1}} \ldots \partial x_{n}^{\alpha_{n}}} .
$$


Here and below we assume summation over repeated indices. The index $i$ runs from 1 to $n$ and the index $a$ runs from 1 to $m$.

Thus, for the functions $F: J^{p}\left(\mathbb{R}^{n}, \mathbb{R}^{m}\right) \rightarrow \mathbb{R}$ we have

$$
D_{i} F=\frac{\partial F}{\partial x_{i}}+u_{\alpha+\delta_{i}}^{a} \frac{\partial F}{\partial u_{\alpha}^{a}},
$$

where the summation over $\alpha$ is over all $n$-tuples $\left(\alpha_{1}, \ldots, \alpha_{n}\right)$ of length $|\alpha| \leqslant p$. The sum is well defined since $F$ is a function of only a finite number of arguments.

\section{Example 2.2}

The space on which second-order ordinary differential equations are defined is $J^{2}(\mathbb{R}, \mathbb{R})$. The underlying space is $\mathbb{R} \times \mathbb{R}$ with local coordinates $(x, y)$. The local coordinates on $J^{2}(\mathbb{R}, \mathbb{R})$ are then $\left(x, y, y^{\prime}, y^{\prime \prime}\right)$ and the total derivative with respect to $x$ is

$$
D_{x}=\frac{\partial}{\partial x}+y^{\prime} \frac{\partial}{\partial y}+y^{\prime \prime} \frac{\partial}{\partial y^{\prime}}+y^{\prime \prime \prime} \frac{\partial}{\partial y^{\prime \prime}} .
$$

\section{Example 2.3}

For $J^{2}\left(\mathbb{R}^{2}, \mathbb{R}\right)$ we have the underlying space $\mathbb{R}^{2} \times \mathbb{R}$ with local coordinates $(x, y, u)$ and the local coordinates on $J^{2}\left(\mathbb{R}^{2}, \mathbb{R}\right)$ are then $\left(x, y, u, u_{x}, u_{y}, u_{x x}, u_{x y}, u_{y y}\right)$. We have two total derivatives

$$
\begin{aligned}
D_{x}= & \frac{\partial}{\partial x}+u_{x} \frac{\partial}{\partial u}+u_{x x} \frac{\partial}{\partial u_{x}}+u_{x y} \frac{\partial}{\partial u_{y}}+u_{x x x} \frac{\partial}{\partial u_{x x}}+u_{x x y} \frac{\partial}{\partial u_{x y}} \\
& +u_{x y y} \frac{\partial}{\partial u_{y y}}, \\
D_{y}= & \frac{\partial}{\partial y}+u_{y} \frac{\partial}{\partial u}+u_{x y} \frac{\partial}{\partial u_{x}}+u_{y y} \frac{\partial}{\partial u_{y}}+u_{y y y} \frac{\partial}{\partial u_{y y}}+u_{y y x} \frac{\partial}{\partial u_{x y}} \\
& +u_{x x y} \frac{\partial}{\partial u_{x x}} .
\end{aligned}
$$

The total derivatives presented in these examples are instances of generalized vector fields rather than vector fields since the coefficient $y^{\prime \prime \prime}$ and $u_{x x x}, u_{x x y}, u_{y y y}$ appear, and these are coordinates on the jet bundles $J^{3}(\mathbb{R}, \mathbb{R})$ and $J^{3}\left(\mathbb{R}^{2}, \mathbb{R}\right)$. This problem can be circumvented by working on the infinite jet bundle $J^{\infty}\left(\mathbb{R}^{n}, \mathbb{R}^{m}\right)$ where the local coordinates include derivatives $u_{\alpha}^{a}$ of arbitrary order (that is, with $|\alpha|$ taking on all possible positive integer values), and in this case the operators $D_{i}$ mentioned above are vector fields. Functions on this infinite jet bundle are defined as being 
functions of only a finite nonzero number of arguments. A rigorous definition is given in [41, but we shall not need this construction. We do not use jet bundles other than in an informal way and the total derivatives are to be understood as generalized vector fields.

\subsection{Lie symmetries of differential equations}

In this section we are interested in point symmetries of differential equations. Given a system of differential equations $\mathcal{L}$, a point symmetry of $\mathcal{L}$ is a local diffeomorphism acting on the space of independent and dependent variables that maps solutions of the system $\mathcal{L}$ to solutions of the same system (see for instance [6], [33]). In general, a symmetry of $\mathcal{L}$ is a transformation of functions that maps any solution of $\mathcal{L}$ to a solution of the same system. Note that according to this, a general symmetry may be a transformation which involves derivatives, integrals, etc., whereas a point symmetry is a transformation on the underlying space of a differential equation (that is, on the space of independent and dependent variables). In the following, we refer to point symmetries as just symmetries.

We begin with a definition of what we mean by a system of differential equations $([33])$ :

Definition 2.3. A system $\mathcal{L}$ of $l$ differential equations of order $p$ for $m$ unknown functions $\left(u^{1}, \ldots, u^{m}\right)$ in $n$ independent variables $\left(x_{1}, \ldots, x_{n}\right)$ is a system of equations

$$
L\left(x, u_{(p)}\right)=0 \quad \text { with a differential function } L: J^{p}\left(\mathbb{R}^{n}, \mathbb{R}^{m}\right) \rightarrow \mathbb{R}^{l},
$$

where $p, m, n$ and $l$ are positive integers. This system is of maximal rank if the smooth function $L: J^{p}\left(\mathbb{R}^{n}, \mathbb{R}^{m}\right) \rightarrow \mathbb{R}^{l}$ has rank $l$, that is, the differential $\mathrm{d} L$ mapping the tangent space at each point of the domain of $L$ to $\mathbb{R}^{l}$ has rank $l$.

\section{Example 2.4}

A system of two equations of order two of one function of independent variables $\left(x_{1}, x_{2}\right)$ is a smooth map $L: J^{2}\left(\mathbb{R}^{2}, \mathbb{R}\right) \rightarrow \mathbb{R}^{2}$ such that

$$
\mathcal{L}:\left\{\begin{array}{l}
L^{1}\left(x, u_{(2)}\right)=0 \\
L^{2}\left(x, u_{(2)}\right)=0
\end{array}\right.
$$

For illustration one can take $\mathcal{L}$ to be the system

$$
L^{1}: u_{t}-u_{x x}-3 u_{x}-u=0, \quad \text { and } \quad L^{2}: u_{t t}-u_{x}-2 u_{x x}=0 .
$$

The differential $\mathrm{d} L$ is then easily computed to be

$$
\mathrm{d} L:=\left(\frac{\partial L^{\nu}}{\partial x_{i}}, \frac{\partial L^{\nu}}{\partial u_{\alpha}^{a}}\right)=\left(\begin{array}{cccccccc}
0 & 0 & -1 & 1 & -3 & 0 & 0 & -1 \\
0 & 0 & 0 & 0 & -1 & 1 & 0 & -2
\end{array}\right),
$$

which is of rank two whenever $L\left(x, u_{(2)}\right)=0$. 
Definition 2.4. A symmetry transformation of a system $\mathcal{L}$ of $l$ differential equations

$$
L\left(x, u_{(p)}\right)=0 \quad \text { with a differential function } L: J^{p}\left(\mathbb{R}^{n}, \mathbb{R}^{m}\right) \rightarrow \mathbb{R}^{l},
$$

is a smooth, invertible map

$$
\Phi: \mathbb{R}^{n} \times \mathbb{R}^{m} \rightarrow \mathbb{R}^{n} \times \mathbb{R}^{m}, \quad \Phi(x, u)=(\tilde{x}, \tilde{u})
$$

so that $L\left(\tilde{x}, \tilde{u}_{(p)}\right)=0$ whenever $L\left(x, u_{(p)}\right)=0$.

Any symmetry $\Phi$ will induce an action on the derivatives of a function. This action is defined as follows:

Definition 2.5. The map $\left(x, u_{(p)}\right) \rightarrow\left(\tilde{x}, \tilde{u}_{(p)}\right)$ induced by the map $\Phi:(x, u) \rightarrow$ $(\tilde{x}, \tilde{u})$ is called the pth prolongation of $\Phi$, denoted by $\Phi_{(p)}$.

It is an essentially cumbersome task to find symmetries of differential equations with Definition 2.4. However, using this definition we can restrict our attention to (locally defined) one-parameter groups generated by vector fields of the form

$$
X=\xi^{i}(x, u) \partial_{x_{i}}+\eta^{a}(x, u) \partial_{u^{a}} .
$$

The action of this vector field can be extended to an action on functions on the jet bundle $P: J^{p}\left(\mathbb{R}^{n}, \mathbb{R}^{m}\right) \rightarrow \mathbb{R}$. This extended action is called the $p$ th prolongation of the vector field $X$, and is denoted by $X_{(p)}$ and is defined by the formula

$$
X_{(p)}=\xi^{i}(x, u) \partial_{x_{i}}+\eta^{a}(x, u) \partial_{u^{a}}+\eta_{\alpha}^{a} \partial_{u_{\alpha}^{a}}
$$

The sum over $\alpha$ is over all $n$-tuples $\alpha$ with $1 \leqslant|\alpha| \leqslant p$. The coefficients $\eta^{a, \alpha}$ are defined as follows for each $\alpha=\left(\alpha_{1}, \ldots, \alpha_{n}\right)$ :

$$
\eta^{a, \alpha}=D^{\alpha}\left(\eta^{a}-\xi^{i} u_{i}^{a}\right)+\xi^{i} u_{\alpha+\delta_{i}}^{a}
$$

with $D^{\alpha}=D_{1}^{\alpha_{1}} \cdots D_{n}^{\alpha_{n}}$. Alternatively, they can may be given recursively as

$$
\eta^{a, \delta_{i}}=D_{i} \eta^{a}-u_{i^{\prime}}^{a} D_{i} \xi^{i^{\prime}}, \quad \eta^{a, \alpha+\delta_{i}}=D_{i} \eta^{a, \alpha}-u_{\alpha+\delta_{i^{\prime}}}^{a} D_{i} \xi^{i^{\prime}}
$$

for $|\alpha| \geqslant 1$.

\section{Example 2.5}

For the vector field

$$
X=x \partial_{x}+2 u \partial_{u}
$$

defined on the underlying space $\mathbb{R} \times \mathbb{R}$, the first prolongation $X_{(1)}$ is

$$
X_{(1)}=x \partial_{x}+2 u \partial_{u}+\eta^{x} \partial_{u_{x}}=x \partial_{x}+2 u \partial_{u}+u_{x} \partial_{u_{x}}
$$

since $\eta^{x}=D_{x}\left(2 u-x u_{x}\right)+x u_{x x}=u_{x}$. 


\section{Example 2.6}

The vector fields $X=\partial_{x}, Y=x \partial_{x}, Z=x^{2} \partial_{x}$ on the underlying space $\mathbb{R}^{2} \times \mathbb{R}$ span a realization of the Lie algebra $\operatorname{sl}(2, \mathbb{R})$. The commutation relations are $[Y, X]=-X,[Y, Z]=Z$ and $[X, Z]=2 Y$. The second prolongations of these vector fields are

$$
\begin{aligned}
& X_{(2)}=\partial_{x}, \quad Y_{(2)}=x \partial_{x}-u_{x} \partial_{u_{x}}-2 u_{x x} \partial_{u_{x x}} \\
& Z_{(2)}=x^{2} \partial_{x}-2 x u_{x} \partial_{u_{x}}-\left(2 u_{x}+4 x u_{x x}\right) \partial_{u_{x x}}
\end{aligned}
$$

For more details on these definitions and examples, we refer to [33].

Lemma 2.1. Let $\Phi_{X}(t)$ denote the local flow of a vector field $X$ on $\mathbb{R}^{n} \times \mathbb{R}^{m}$ and $\Phi_{X_{(p)}}(t)$ the local flow of $X_{(p)}$ on $J^{p}\left(\mathbb{R}^{n}, \mathbb{R}^{m}\right)$, then

$$
\left(\Phi_{X}(t)\right)_{(p)}=\Phi_{X_{(p)}}(t) .
$$

For a proof, see [33].

We can now define what we mean by a Lie symmetry vector field for a system of differential equations:

Definition 2.6. A vector field $X$ defined by

$$
X=\xi^{i}(x, u) \partial_{x_{i}}+\eta^{a}(x, u) \partial_{u^{a}}
$$

is an infinitesimal point symmetry of a system $\mathcal{L}$ of differential equations

$$
L\left(x, u_{(p)}\right)=0, \quad \text { where } \quad L: J^{p}\left(\mathbb{R}^{n}, \mathbb{R}^{m}\right) \rightarrow \mathbb{R}^{l},
$$

if each local diffeomorphism from the local flow $\Phi_{X}(t)$ generated by $X$ is a symmetry of the system $\mathcal{L}$.

The basic result on Lie symmetries of differential equations is the following:

Theorem 2.1. If the (smooth) vector field $X$ defined on the underlying space $\mathbb{R}^{n} \times \mathbb{R}^{m}$ is a Lie symmetry of the system $\mathcal{L}$ of differential equations $L\left(x, u_{(p)}\right)=0$, where $L: J^{p}\left(\mathbb{R}^{n}, \mathbb{R}^{m}\right) \rightarrow \mathbb{R}^{l}$ is of maximal rank, then

$$
X_{(p)} L=0 \quad \text { whenever } \quad L\left(x, u_{(p)}\right)=0 .
$$

If $X, Y$ are two infinitesimal point symmetries of the system $\mathcal{L}$, then so is their commutator $[X, Y]$. Here $X_{(p)} L=0$ means $X_{(p)} L^{q}=0$ for all $q=1, \ldots, l$.

Note that this result only says that if $\Phi_{X}(t)$, at least for $t \in I$ for some interval $I$ of zero, is a symmetry, then $X_{(p)} L=0$. It requires that $\Phi_{X}(t) u$ to be a solution of $L\left(x, u_{(p)}\right)=0$ whenever $u$ is a solution. The converse statement requires the extra condition of nondegeneracy. 


\subsubsection{Nondegenerate systems of differential equations}

Our work deals with nondegenerate systems of differential equations. We have the following definitions (see [33]):

Definition 2.7. A system $\mathcal{L}$ of $p$ th order differential equations $L\left(x, u_{(p)}\right)=0$ is said to be locally solvable at the point $\left(x, u_{(p)}\right)$ for which $L\left(x, u_{(p)}\right)=0$ if there exists a smooth solution $u=f(x)$ of the system $\mathcal{L}\left(x, u_{(p)}\right)=0$ which is defined in some neighborhood of $x$ and satisfying $u_{(p)}=f(x)_{(p)}$, where $f(x)_{(p)}$ denotes the collection of all derivatives of $f(x)$ of order up to $p$.

It is locally solvable if it is locally solvable at every point of the set

$$
\left\{\left(x, u_{(p)}\right): L\left(x, u_{(p)}\right)=0\right\} .
$$

Definition 2.8. A system of differential equations is said to be non-degenerate if it is locally solvable and of maximal rank at each point of the set

$$
\left\{\left(x, u_{(p)}\right): L\left(x, u_{(p)}\right)=0\right\} .
$$

The importance of non-degeneracy is illustrated in the following theorem:

Theorem 2.2. Suppose $L\left(x, u_{(p)}\right)=0$ is a nondegenerate system of differential equations. A vector field $X$ on the underlying space $\mathbb{R}^{n} \times \mathbb{R}^{m}$ is an infinitesimal symmetry of this system if and only if

$$
X_{(p)} L=0 \quad \text { whenever } \quad L\left(x, u_{(p)}\right)=0 .
$$

For a proof, see 33$]$. 


\section{Classes of differential equations and equivalence transformations}

We give here a short exposition of the main technical concepts that are used in our approach to the group classification of differential equations: these are the idea of a class of differential equations and equivalence transformations and concepts related to them.

\subsection{Classes of differential equations}

To introduce the concept of a class of differential equations we look at the example of an evolution equation of second order:

$$
u_{t}=F\left(t, x, u, u_{x}, u_{x x}\right),
$$

where $F$ is a smooth function of its arguments, and it belongs to a class of equations with the following conditions on $F$

$$
F_{u_{t}}=F_{u_{t x}}=F_{u_{t t}}=0, \quad F_{u_{x x}} \neq 0 .
$$

We call $F$ an arbitrary element of the system

$$
u_{t}=F\left(t, x, u, u_{x}, u_{x x}\right), \quad F_{u_{t}}=F_{u_{t x}}=F_{u_{t t}}=0, \quad F_{u_{x x}} \neq 0 .
$$

This can be symbolized by

$$
L_{\theta}\left(t, x, u_{(2)}, \theta\left(t, x, u_{(2)}\right)\right)=0,
$$

where $\theta=F$ and belongs to the set of (locally) smooth functions satisfying

$$
\theta_{u_{t}}=\theta_{u_{t x}}=\theta_{u_{t t}}=0, \quad \theta_{u_{x x}} \neq 0 .
$$


The functions $\theta$ are called arbitrary elements. Note that in a system of differential equations, there will in general be more than one arbitrary element.

In our work we follow [33], and we consider systems of differential equations

$$
\mathcal{L}_{\theta}: \quad L\left(x, u_{(p)}, \theta_{(q)}\left(x, u_{(p)}\right)\right)=0,
$$

parameterized by the tuple of arbitrary elements $\theta\left(x, u_{(p)}\right)$, where $\theta$ denotes the tuple $\theta=\left(\theta^{1}, \ldots, \theta^{k}\right)$ and $\theta_{(q)}$ is the set of derivatives of $\theta$ up to and including order $q$. This tuple of arbitrary elements $\theta$ satisfies an auxiliary system of equations $\mathcal{S}$ which consists of equations of the form $S\left(x, u_{(p)}, \theta_{(q)}\left(x, u_{(p)}\right)\right)=0$. This auxiliary system may also be augmented by some non-vanishing condition written representatively as $\Sigma\left(x, u_{(p)}, \theta_{(q)}\left(x, u_{(p)}\right)\right) \neq 0$, which is taken to mean that no component of $\Sigma$ vanishes.

In this way, we have the class of systems of differential equations $\mathcal{L}_{\theta}$ where the arbitrary elements $\theta$ run through $\mathcal{S}$. We denote this class by $\left.\mathcal{L}\right|_{\mathcal{S}}$.

\section{Example 3.1}

In the above example of the class of second order evolution equation we have: $n=2, m=1, p=2, l=1, \theta=F$, the auxiliary system of the class is $\mathcal{S}$ : $\theta_{u_{t}}=\theta_{u_{t x}}=\theta_{u_{t t}}=0$ and the non-vanishing condition is $\theta_{u_{x x}} \neq 0$.

\section{Example 3.2}

Consider the class of second order nonlinear wave equations of the form

$$
u_{t t}=f\left(x, u_{x}\right) u_{x x}+g\left(x, u_{x}\right),
$$

where the arbitrary elements $f$ and $g$ depend on $x$ and $u_{x}$. We have a single system with two arbitrary elements $\theta^{1}=f$ and $\theta^{2}=g$ depending on $x$ and $u_{x}$. The auxiliary system associated to this class is formed by the equations:

$$
\begin{aligned}
& \theta_{t}^{1}=\theta_{u}^{1}=\theta_{u_{t}}^{1}=\theta_{u_{t x}}^{1}=\theta_{u_{x x}}^{1}=\theta_{u_{t t}}^{1}=0, \\
& \theta_{t}^{2}=\theta_{u}^{2}=\theta_{u_{t}}^{2}=\theta_{u_{t x}}^{2}=\theta_{u_{x x}}^{2}=\theta_{u_{t t}}^{2}=0 .
\end{aligned}
$$

The nonvanishing conditions are $\theta^{1} \neq 0$ and $\left(\theta_{u_{x}}^{1}, \theta_{u_{x} u_{x}}^{2}\right) \neq(0,0)$.

\section{Example 3.3}

For the class of linear Schrödinger equations $i \psi_{t}+\psi_{x x}+V(t, x) \psi=0$, we have a single arbitrary element $\theta=V$ with the auxiliary system of the form

$$
\begin{aligned}
& \theta_{\psi}=\theta_{\psi^{*}}=\theta_{\psi_{t}}=\theta_{\psi_{t}^{*}}=\theta_{\psi_{x}}=\theta_{\psi_{x}^{*}}=0, \\
& \theta_{\psi_{t x}}=\theta_{\psi_{t x}^{*}}=\theta_{\psi_{t t}}=\theta_{\psi_{t t}^{*}}=\theta_{\psi_{x x}}=\theta_{\psi_{x x}^{*}}=0 .
\end{aligned}
$$




\subsection{Admissible transformations}

In this section we look at admissible transformations of classes of differential equations and some of their properties and its generalizations. More details can be found in [37, [40].

If we take two systems of equations $\mathcal{L}_{\theta}$ and $\mathcal{L}_{\tilde{\theta}}$ from a given class $\left.\mathcal{L}\right|_{\mathcal{S}}$, then the elements $\theta, \tilde{\theta}$ may or may not be equivalent under some invertible transformation. For instance, the evolution equations

$$
u_{t}=u^{2}\left[\frac{u_{3}}{u_{1}^{3}}-\frac{3}{2} \frac{u_{2}^{2}}{u_{1}^{4}}\right], \quad u_{t}=\left[\frac{u_{3}}{u_{1}^{3}}-\frac{3}{2} \frac{u_{2}^{2}}{u_{1}^{4}}\right]
$$

cannot be mapped into one another by any equivalence transformation of the class of third-order evolution equations of the form $u_{t}=F\left(t, x, u, u_{1}, u_{2}\right) u_{3}+$ $G\left(t, x, u, u_{1}, u_{2}\right)$. Thus we are led to the concept of admissible transformations: that is, transformations that map $\mathcal{L}_{\theta}$ to $\mathcal{L}_{\tilde{\theta}}$.

Definition 3.1. If $\mathcal{L}_{\theta}$ and $\mathcal{L}_{\tilde{\theta}}$ are two systems belonging to the class $\left.\mathcal{L}\right|_{\mathcal{S}}$, we say an admissible transformation from $\mathcal{L}_{\theta}$ to $\mathcal{L}_{\tilde{\theta}}$ is an invertible point transformation of the underlying space having local coordinates $(x, u)$ which maps $\mathcal{L}_{\theta}$ to $\mathcal{L}_{\tilde{\theta}}$. We denote by $T(\theta, \tilde{\theta})$ the set of point transformations from $\mathcal{L}_{\theta}$ to $\mathcal{L}_{\tilde{\theta}}$.

Definition 3.2. We define the set of admissible transformations in the class of differential equations as the set of triples

$$
\{(\theta, \tilde{\theta}, \varphi) \mid \theta, \tilde{\theta} \in \mathcal{S}, \varphi \in \mathrm{T}(\theta, \tilde{\theta})\} .
$$

We note (see [23]) that the admissible transformations for the class $\left.\mathcal{L}\right|_{\mathcal{S}}$ have the structure of a groupoid: the identity transformation is quite obviously an admissible transformation; if $\varphi \in \mathrm{T}\left(\theta_{1}, \theta_{2}\right)$ and $\psi \in \mathrm{T}\left(\theta_{3}, \theta_{4}\right)$ then the composition $\psi \circ \varphi$ is defined only if $\theta_{2}=\theta_{3}$ and then $\psi \circ \varphi \in \mathrm{T}\left(\theta_{1}, \theta_{4}\right)$. The associativity of composition is inherited from the associativity of point transformations: $\varphi \circ(\psi \circ$ $\rho)=(\varphi \circ \psi) \circ \rho$ whenever the composition is defined. We are thus led to the following definition:

Definition 3.3. The set of all admissible transformations with the groupoid structure described above is called the equivalence groupoid of the class $\left.\mathcal{L}\right|_{\mathcal{S}}$ and is denoted by $\mathcal{G}^{\sim}$. Thus, $\mathcal{G}^{\sim}=\mathcal{G}^{\sim}\left(\left.\mathcal{L}\right|_{\mathcal{S}}\right):=\{(\theta, \tilde{\theta}, \varphi) \mid \theta, \tilde{\theta} \in \mathcal{S}, \varphi \in \mathrm{T}(\theta, \tilde{\theta})\}$.

The equivalence groupoid $\mathcal{G}^{\sim}$ is computed by the use of the direct method. To do this, we fix two arbitrary systems $\mathcal{L}_{\theta}: L\left(x, u_{(p)}, \theta_{(q)}\left(x, u_{(p)}\right)\right)=0$, and $\mathcal{L}_{\tilde{\theta}}$ : $L\left(\tilde{x}, \tilde{u}_{(p)}, \tilde{\theta}_{(q)}\left(\tilde{x}, \tilde{u}_{(p)}\right)\right)=0$, and search for invertible point transformations,

$$
\varphi: \tilde{x}_{i}=X^{i}(x, u), \quad \tilde{u}^{a}=u^{a}(x, u), \quad i=1, \ldots, n ; \quad a=1, \ldots, m,
$$

that connect the two systems. Using the chain rule we extend these transformations to transformations of derivatives, expressing the derivatives of tilded variables in terms of untilded ones. In this way we obtain a transformed system which should be satisfied identically when substituted into the system $\mathcal{L}_{\theta}$. This substitution leads to the system of determining equations for transformational components 
of $\varphi$. Solving these determining equations gives the required $\mathcal{G}^{\sim}$. A particular case of such transformations is that of symmetries: they map a given system $\mathcal{L}_{\theta}$ to itself. The set of all such symmetries is denoted by $G_{\theta}$.

Definition 3.4. The maximal point symmetry group of the system of differential equations $\mathcal{L}_{\theta}$ for a fixed $\theta \in \mathcal{S}$ is the group $G_{\theta}$ of transformations in the space of variables $(x, u)$ such that any solution of the system $\mathcal{L}_{\theta}$ is mapped to a solution of the same system. That is $G_{\theta}:=\mathrm{T}(\theta, \theta)$.

The intersection of the maximal point symmetry groups of all systems from this class is called the kernel group, $G^{\cap}=G^{\cap}\left(\left.\mathcal{L}\right|_{\mathcal{S}}\right):=\bigcap_{\theta \in \mathcal{S}} G_{\theta}$.

The generators of one-parameter subgroups of the groups $G_{\theta}$ and $G^{\cap}$ form the Lie algebras $\mathfrak{g}_{\theta}$ and $\mathfrak{g}^{\bigcap}$, respectively, and are called the maximal Lie invariance algebra of the system $\mathcal{L}_{\theta}$ and the kernel invariance algebra of the class $\left.\mathcal{L}\right|_{\mathcal{S}}$.

\subsection{Equivalence groups}

The notion of equivalence group, developed in more detail in [37, [40] plays an important role in the group classification of differential equations. In this section we look at the equivalence group of the class of differential equations $\left.\mathcal{L}\right|_{\mathcal{S}}$, some of its properties and and its generalization.

\subsubsection{Usual equivalence group and equivalence algebra}

Definition 3.5. The usual equivalence group $G^{\sim}$ of the class $\left.\mathcal{L}\right|_{\mathcal{S}}$ is the set of transformations $\mathcal{T}$ satisfying the following properties:

1. $\mathcal{T}$ is a point transformation in the space $J^{p}\left(\mathbb{R}^{n}, \mathbb{R}^{m}\right) \times \mathcal{S}$ endowed with local coordinates $\left(x, u_{(p)}, \theta\right)$, which is projectable to the space $J^{q}\left(\mathbb{R}^{n}, \mathbb{R}^{m}\right)$ with local coordinates $\left(x, u_{(q)}\right)$ for each $0 \leqslant q \leqslant p$, so that $\left.\mathcal{T}\right|_{\left(x, u_{(q)}\right)}$ is the $q$ th order prolongation of $\left.\mathcal{T}\right|_{(x, u)}$.

2. $\mathcal{T}$ maps every system from the class $\left.\mathcal{L}\right|_{\mathcal{S}}$ to a system from the same class.

Remark 3.1. In Definition 3.5 the notation $\left.\mathcal{T}\right|_{\left(x, u_{(q)}\right)}$ means the restriction of $\mathcal{T}$ to the space $J^{q}\left(\mathbb{R}^{n}, \mathbb{R}^{m}\right)$. The admissible transformations of the form $\left(\theta, \tilde{\theta},\left.\mathcal{T}\right|_{(x, u)}\right)$, where $\theta, \mathcal{T} \theta \in \mathcal{S}$ and $\mathcal{T} \in G^{\sim}$, are said to be induced by the equivalence transformation $\mathcal{T}$.

\section{Example 3.4}

For the class of equations $i \psi_{t}+\psi_{x x}+V(t, x) \psi=0$, where $\psi$ is unknown complexvalued function of $(t, x)$ and $V$ is an arbitrary complex-valued smooth function depending on its arguments, the point transformations $\tilde{t}=t, \tilde{x}=-x, \tilde{\psi}=\psi$ and $\tilde{V}=V$ is an equivalence transformation. 
Remark 3.2. It is important to note that the entire equivalence group $G^{\sim}$ of the class $\left.\mathcal{L}\right|_{\mathcal{S}}$ is generated by two kinds of transformations: continuous equivalence transformations and discrete equivalence transformations. In most papers scientists looked at continuous equivalence transformations and ignored discrete equivalence transformations whereas later are found also useful in the course of group classification of differential equations.

Both the equivalence groupoid $\mathcal{G}^{\sim}$ and the equivalence group $G^{\sim}$ of the class of differential equations $\left.\mathcal{L}\right|_{\mathcal{S}}$ play a crucial role in the group classification of differential equations. The knowledge of the equivalence groupoid $\mathcal{G} \sim$ of the class $\left.\mathcal{L}\right|_{\mathcal{S}}$ simplifies the process of classifying Lie symmetry extensions of this class. Using the equivalence groupoid $\mathcal{G}^{\sim}$, we can easily obtain the equivalence group $G^{\sim}$ of the class $\left.\mathcal{L}\right|_{\mathcal{S}}$. The group classification of the class under study is easily investigated once these objects are related in a nice way. Properties of classes of differential equations associated with the relation between these objects are discussed in the Section 3.4

We close this section with a description of the set of generators of the group $G^{\sim}$.

Definition 3.6. We call an equivalence algebra of the class $\left.\mathcal{L}\right|_{\mathcal{S}}$ denoted by $\mathfrak{g}^{\sim}$, a Lie algebra formed by the generators of one-parameter subgroups of the equivalence group $G^{\sim}$.

It is convenient, for a given class of differential equations, to use the direct method to find the equivalence groupoid $\mathcal{G}^{\sim}$, which is used to construct the equivalence group $G^{\sim}$. Once the latter is known, we can find the infinitesimal generators of $G^{\sim}$, which constitute the algebra $\mathfrak{g}^{\sim}$.

\subsubsection{Generalized and generalized extended equivalence groups}

The notion of equivalence group can be generalized in several ways. For the usual equivalence group, the transformations of independent and dependent variables $(x, u)$ do not depend on the arbitrary element $\theta$. In this case the equivalence transformations are of the form $(\tilde{x}, \tilde{u})=(X(x, u), U(x, u)), \tilde{\theta}=\Theta(x, u, \theta)$. The class $\left.\mathcal{L}\right|_{\mathcal{S}}$, with usual equivalence group $G^{\sim}$, may have other equivalence transformations which do not belong to $G^{\sim}$ and, together with this group, form different variations of equivalence groups [40, [44].

If the transformations of the variables $(x, u)$ depend on the arbitrary element $\theta$ then the equivalence group is called the generalized equivalence group and is denoted by $G_{\text {gen }}^{\sim}$. More specifically, this means that any $\mathcal{T} \in G_{\text {gen }}^{\sim}$ is a point transformation in the space $(x, u, \theta)$ such that

$$
\forall \theta \in \mathcal{S}: \quad \mathcal{T} \theta \in \mathcal{S} \quad \text { and }\left.\quad \mathcal{T}(\cdot, \cdot, \theta(\cdot, \cdot))\right|_{(x, u)} \in \mathrm{T}(\theta, \mathcal{T} \theta)
$$

This is equivalent to saying that the generalized equivalence transformations are of the form $(\tilde{x}, \tilde{u}, \tilde{\theta})=(X, U, \Theta)(x, u, \theta)$. When the transformations of the arbitrary elements are expressed in terms of the old arbitrary elements of the class in a nonlocal way (involving the integrals of the arbitrary elements) then the corresponding equivalence group is called the extended equivalence group and is denoted by $\hat{G}^{\sim}$. 
Furthermore, if, in addition, the transformational components of the equivalence transformations (both components for variables $(x, u)$ and arbitrary elements) are expressed in terms of the arbitrary elements of the class in a non-local way then the equivalence group is said to be the generalized extended equivalence group and it is denoted by $\hat{G}_{\mathrm{gen}}^{\sim}$. Different types of these equivalence groups are discussed in [22], 44].

\section{Example 3.5}

Consider the class of variable coefficient diffusion-convection equations of the form

$$
f(x) u_{t}=\left(g(x) A(u) u_{x}\right)_{x}+h(x) B(u) u_{x},
$$

where $f=f(x), g=g(x), h=h(x), A=A(u)$ and $B=B(u)$ are arbitrary smooth functions of their variables, $f g h A \neq 0$ and $\left(A_{u}, B_{u}\right) \neq(0,0)$ studied in [22]. The usual equivalence group $G^{\sim}$ of this class consists of the point transformations of the form:

$$
\begin{aligned}
& \tilde{t}=\sigma_{1} t+\sigma_{2}, \quad \tilde{x}=X(x), \quad \tilde{u}=\sigma_{3} u+\sigma_{4}, \\
& \tilde{f}=\frac{\varepsilon_{1} \sigma_{1}}{X_{x}} f, \quad \tilde{g}=\varepsilon_{1} \varepsilon_{2}{ }^{-1} X_{x} g, \quad \tilde{h}=\varepsilon_{1} \varepsilon_{3}{ }^{-1}, \\
& \tilde{A}=\varepsilon_{2} A, \quad \tilde{B}=\varepsilon_{3} B,
\end{aligned}
$$

where $\sigma_{j}(j=1, \ldots, 4)$ and $\varepsilon_{i}(i=1, \ldots, 3)$ are arbitrary constants, $\sigma_{1} \sigma_{3} \varepsilon_{1} \varepsilon_{2} \varepsilon_{3} \neq 0$, $X$ an arbitrary smooth function of $x$ with $X_{x} \neq 0$ while the transformations

$$
\begin{aligned}
& \tilde{t}=\sigma_{1} t+\sigma_{2}, \quad \tilde{x}=X(x), \quad \tilde{u}=\sigma_{3} u+\sigma_{4}, \\
& \tilde{f}=\frac{\varepsilon_{1} \sigma_{1} \varphi}{X_{x}} f, \quad \tilde{g}=\varepsilon_{1} \varepsilon_{2}{ }^{-1} X_{x} \varphi g, \quad \tilde{h}=\varepsilon_{1} \varepsilon_{3}{ }^{-1} \varphi h, \\
& \tilde{A}=\varepsilon_{2} A, \quad \tilde{B}=\varepsilon_{3}\left(B+\varepsilon_{4} A\right),
\end{aligned}
$$

where $\sigma_{j}(j=1, \ldots, 4)$ and $\varepsilon_{i}(i=1, \ldots, 4)$ are arbitrary constants, $\sigma_{1} \sigma_{3} \varepsilon_{1} \varepsilon_{2} \varepsilon_{3} \neq 0$, $X$ an arbitrary smooth function of $x$ with $X_{x} \neq 0$,

$$
\varphi=\exp \left(-\varepsilon_{4} \int \frac{h(x)}{g(x)} \mathrm{d} x\right),
$$

constitute an extended equivalence group $\hat{G}^{\sim}$.

\section{Example 3.6}

The equivalence transformations corresponding to the class of variable-coefficient Korteweg de-Vries equations of the form $u_{t}+f(t, x) u u_{x}+g(t, x) u_{x x x}=0$ with $f g \neq 0$ studied in [44] whose form is

$$
\tilde{t}=T(t) \quad \tilde{x}=\frac{\sigma_{1} x+\sigma_{2}}{\varphi(t)}+\sigma_{5}, \quad \tilde{u}=\varphi(t) u-\sigma_{3} x-\frac{\sigma_{2} \sigma_{3}}{\sigma_{1}},
$$




$$
\tilde{f}(\tilde{t})=\frac{\sigma_{1}}{T_{t}(\varphi(t))^{2}} f(t), \quad \tilde{g}(\tilde{t}, \tilde{x})=\frac{\sigma_{1}^{3}}{T_{t}(\varphi(t))^{3}} g(t, x),
$$

where $T$ is an arbitrary function of $t$ with $T_{t} \neq 0, \varphi=\sigma_{3} \int f(t) \mathrm{d} t+\sigma_{4}$ and $\sigma_{j}(j=1, \ldots, 5)$ are arbitrary constants such that $\sigma_{1}\left(\sigma_{3}^{2}+\sigma_{4}^{2}\right) \neq 0$ constitute a generalized extended equivalence group $\hat{G}_{\text {gen }}^{\sim}$ for that class.

\subsubsection{Gauge equivalence group}

We continue here the discussion of different type of equivalence transformations for the given class $\left.\mathcal{L}\right|_{\mathcal{S}}$. Here we are concerned with equivalence transformations which act only on the arbitrary elements and preserve the space of variables.

Definition 3.7. The equivalence transformations from the group $G^{\sim}$ which act only on the arbitrary elements (and do not change the systems of differential equations), generate gauge admissible transformations. Equivalence transformations of this form are considered as trivial (they are "gauges") and are called gauge equivalence transformations. They constitute the gauge (normal) subgroup $G^{g \sim}$, $G^{g \sim}=\left\{\mathcal{T} \in G^{\sim} \mid \mathcal{T} x=x, \mathcal{T} u=u, \mathcal{T} \theta \sim \theta\right\}$ of the equivalence group $G^{\sim}$. As in the previous consideration, we may have different equivalence groups and also their corresponding gauge subgroups. The values of the arbitrary elements $\theta$ and $\tilde{\theta}$ in $\mathcal{S}$ are gauge equivalent if the systems $\mathcal{L}_{\theta}$ and $\mathcal{L}_{\tilde{\theta}}$ are the same systems of differential equations. Here their sets of solutions coincide.

\section{Example 3.7}

Consider the class $\mathcal{V}$ of nonlinear Schrödinger equations with potentials and modular nonlinearity of the form

$$
i \psi_{t}+\Delta \psi+f(|\psi|) \psi+V(t, x) \psi=0, \quad f_{|\psi|} \neq 0
$$

and consider an equation $\mathcal{L}_{\theta}, \theta=(f, V)$, from this class for any fixed $\theta$. Then the equivalence transformation

$$
\begin{gathered}
\tilde{t}=t, \quad \tilde{x}=x, \quad \tilde{\psi}=\psi, \\
\tilde{f}=f+\beta, \quad \tilde{V}=V-\beta
\end{gathered}
$$

with an arbitrary complex number $\beta$, which maps an equation $\mathcal{L}_{\theta}$ to an equation $\mathcal{L}_{\tilde{\theta}}: i \psi_{t}+\Delta \psi+\tilde{f}(|\psi|) \psi+\tilde{V}(t, x) \psi=0$, does not change the variables but they change only the arbitrary elements. At the same time the initial equation $\mathcal{L}_{\theta}$ coincides with the transformed equation $\mathcal{L}_{\tilde{\theta}}$. Thus, this transformation is a gauge equivalence transformation of the class $\mathcal{V}$. 


\subsubsection{Conditional equivalence groups}

We look at the structure of the equivalence transformations arising from the classes and their subclasses that are singled out from these larger classes by imposing some conditions on the set of arbitrary elements. For more details the reader is referred to 40 .

Consider the class $\left.\mathcal{L}\right|_{\mathcal{S}}$ whose equivalence group $G^{\sim}$ is known and let $\left.\mathcal{L}\right|_{\mathcal{S}^{\prime}}$ be a subclass singled out from the class $\left.\mathcal{L}\right|_{\mathcal{S}}$ by imposing additional constraints on the sets $\mathcal{S}^{\prime}$ and $\Sigma^{\prime}$ of the form $\mathcal{S}^{\prime}\left(x, u_{(p)}, \theta_{\left(q^{\prime}\right)}\right)=0, \quad \Sigma^{\prime}\left(x, u_{(p)}, \theta_{\left(q^{\prime}\right)}\right) \neq 0$ with respect to the arbitrary elements $\theta=\theta\left(x, u_{(p)}\right)$, where $\mathcal{S}^{\prime} \subset \mathcal{S}$ is the set of solution of the united system $\mathcal{S}=0, \Sigma \neq 0, \mathcal{S}^{\prime}=0, \Sigma^{\prime} \neq 0$.

Definition 3.8. The equivalence group $G^{\sim}$ of the class $\left.\mathcal{L}\right|_{\mathcal{S}^{\prime}}, G^{\sim}\left(\left.\mathcal{L}\right|_{\mathcal{S}^{\prime}}\right)$, is called the conditional equivalence group of the whole class $\left.\mathcal{L}\right|_{\mathcal{S}}$ under the conditions $\mathcal{S}^{\prime}=0$ and $\Sigma^{\prime} \neq 0$. The conditional equivalence group is called nontrivial if and only if it is not a subgroup of $G^{\sim}\left(\left.\mathcal{L}\right|_{\mathcal{S}}\right)$. It is said to be maximal under the above conditions on $\mathcal{S}^{\prime}$ and $\Sigma^{\prime}$ if for any subclass $\left.\mathcal{L}\right|_{\mathcal{S}^{\prime \prime}}$ of the class $\left.\mathcal{L}\right|_{\mathcal{S}}$ containing the subclass $\left.\mathcal{L}\right|_{\mathcal{S}^{\prime}}$ we have $G^{\sim}\left(\left.\mathcal{L}\right|_{\mathcal{S}^{\prime}}\right) \nsubseteq G^{\sim}\left(\left.\mathcal{L}\right|_{\mathcal{S}^{\prime \prime}}\right)$. Throughout of this thesis we are concerned with the maximal conditional equivalence groups since they are nontrivial.

\subsection{Normalization properties}

The study of Lie symmetries in the class of differential equations $\left.\mathcal{L}\right|_{\mathcal{S}}$ relies on the properties of point transformations that this class possesses. In this section our aim is to describe the property of normalization with respect to point transformations and we will see throughout the thesis how we can relate the equivalence groupoid, the equivalence group and normalization properties of the concerned class. More details on normalization property can be obtained in [24, 40].

Definition 3.9. A class of differential equations $\left.\mathcal{L}\right|_{\mathcal{S}}$ is said to be normalized (in the usual sense) if the equivalence groupoid $\mathcal{G}^{\sim}$ for this class is generated by its equivalence group $G^{\sim}$. This means that for each triple $(\theta, \tilde{\theta}, \varphi)$ from the equivalence groupoid $\mathcal{G}^{\sim}$, one can find an equivalence transformation $\mathcal{T} \in G^{\sim}$ such that $\tilde{\theta}=\mathcal{T} \theta$ and $\varphi=\left.\mathcal{T}\right|_{(x, u)}$. The class is called normalized in the generalized sense if for any triple $(\theta, \tilde{\theta}, \varphi) \in \mathcal{G}^{\sim}$, there exists an equivalence transformation $\mathcal{T} \in G_{\text {gen }}^{\sim}$ with $\tilde{\theta}=\mathcal{T} \theta$ and $\varphi=\left.\mathcal{T}(x, u, \theta(x, u))\right|_{(x, u)}$.

\section{Example 3.8}

Consider the class of second order ordinary differential equations,

$$
y_{t t}+a_{1}(t) y_{t}+a_{0}(t) y=b(t)
$$

where $a_{1}, a_{0}$ and $b_{0}$ are real-valued arbitrary functions of $t$ and $y=y(t)$ is unknown function of $t$. Its equivalence groupoid $\mathcal{G}^{\sim}$ is constituted by triples $\left(\left(a_{1}, a_{0}, b\right),\left(\tilde{a_{1}}, \tilde{a_{0}}, \tilde{b}\right), \varphi\right)$, where $\varphi$ is a point transformations in the space of variables whose components are

$$
T=T(t), \quad Y=Y^{1}(t) x+Y^{0}(t),
$$


and the transformed arbitrary elements $\left(\tilde{a_{1}}, \tilde{a_{0}}, \tilde{b}\right)$ are given by

$$
\begin{aligned}
& \tilde{a_{1}}=a_{1}-2 \frac{Y_{t}^{1}}{Y^{1}}-\frac{T_{t t}}{T_{t}}, \quad \tilde{a_{0}}=a_{0}+\frac{1}{Y^{1}}\left(T_{t t} Y_{t}^{1}-Y_{t t}^{1}-\tilde{a}_{1} Y_{t}^{1}\right) \\
& \tilde{b}=\frac{1}{T_{t}^{2}}\left(b Y^{1}+Y_{t t}^{0}+\tilde{a}_{1} Y_{t}^{0}-Y_{t}^{0} \frac{T_{t t}}{T_{t}}+\tilde{a}_{0} Y^{0}\right) .
\end{aligned}
$$

Here $T, Y^{1}$ and $Y^{0}$ are real-valued functions of $t$ with $T_{t} Y^{1} \neq 0$. The class 3.2 is normalized and its equivalence group $G^{\sim}$ is constituted by the point transformations of the form (3.3) and (3.4). More generally, the class of homogeneous (resp. inhomogeneous) linear systems of differential equations is normalized.

\section{Example 3.9}

The class of Burgers equations,

$$
u_{t}+u u_{x}+f(t, x) u_{x x}=0, \quad f \neq 0
$$

whose equivalence group $G^{\sim}$ consists of the point transformations of the form

$$
\begin{aligned}
& \tilde{t}=\frac{a_{1} t+a_{0}}{a_{3} t+a_{2}}, \quad \tilde{x}=\frac{\kappa x+\alpha_{1} t+\alpha_{0}}{a_{3} t+a_{2}}, \\
& \tilde{u}=\frac{\kappa\left(a_{3} t+a_{2}\right) u-\kappa a_{3} x+\alpha_{1} a_{2}-\alpha_{0} a_{3}}{a_{1} a_{2}-a_{0} a_{3}}, \quad \tilde{f}=\frac{\kappa^{2}}{a_{1} a_{2}-a_{0} a_{3}} f,
\end{aligned}
$$

where $a_{1}, a_{0}, a_{3}, a_{2}, \alpha_{1}, \alpha_{0}$ and $\kappa$ are constants with $a_{1} a_{2}-a_{0} a_{3} \neq 0$ and $\kappa \neq 0$, is normalized.

From the knowledge of the equivalence groupoid $\mathcal{G}^{\sim}$, one can check by inspection whether or not the class under study is normalized (in the usual sense). It is true when the transformational part $\varphi$ of each admissible transformation does not depend on the fixed initial value $\theta$ of the arbitrary element tuple, and is hence appropriate for an arbitrary initial value of the arbitrary element tuple. Furthermore, the prolongation of $\varphi$ to the space $\left(x, u_{(p)}\right)$ and the further extension to the arbitrary elements according to the relation between the arbitrary elements $\theta$ and $\tilde{\theta}$ give a point transformation in the joint space with local coordinates $\left(x, u_{(p)}, \theta\right)$, see [5]. If the class $\left.\mathcal{L}\right|_{\mathcal{S}}$ is normalized, then the projection of the equivalence algebra of this class contains the linear span of the maximal Lie invariance algebras of equations from the class $\left.\mathcal{L}\right|_{\mathcal{S}}$.

Definition 3.10. A class of differential equation $\left.\mathcal{L}\right|_{\mathcal{S}}$ is called semi-normalized if its equivalence groupoid $\mathcal{G}^{\sim}$ is induced by transformations from its equivalence group $G^{\sim}$ and maximal point symmetry groups $G_{\theta}$ of equations from this class, i.e., for any triple $(\theta, \tilde{\theta}, \varphi) \in \mathcal{G}^{\sim}, \exists \mathcal{T} \in G^{\sim}$ and $\varphi^{1} \in G_{\theta}$ such that $\tilde{\theta}=\mathcal{T} \theta$ and $\varphi=\left.\mathcal{T}\right|_{(x, u)} \circ \varphi^{1}$. 


\section{Example 3.10}

The class of second order ordinary differential equations of the form 3.2 with $b(t)=0$ is semi-normalized. Its equivalence group $G^{\sim}$ consists of point transformations of the form $(3.3)$ and $(3.4)$ with $Y^{0}=0$.

Besides these two properties of the normalization, we define another useful property in the group classification of differential equations: uniform semi-normalization. The theory of uniform semi-normalization, which is new in group classification, is developed in the papers that make up this thesis. Here we give only the definition; the complete theory, together with the Theorem on splitting of symmetry groups in uniformly semi-normalized classes, is presented in the paper [24.

Definition 3.11. Let $\mathcal{G}^{\sim}$ and $G^{\sim}$ be the equivalence groupoid and the (usual) equivalence group for the class $\left.\mathcal{L}\right|_{\mathcal{S}}$. Let for each $\theta \in \mathcal{S}$ the point symmetry group $G_{\theta}$ of the equation $\left.\mathcal{L}_{\theta} \in \mathcal{L}\right|_{\mathcal{S}}$ contains a subgroup $N_{\theta}$ such that the family $\mathcal{N}_{\mathcal{S}}=\left\{N_{\theta} \mid \theta \in \mathcal{S}\right\}$ of all these subgroups satisfies the following properties:

1. $\left.\mathcal{T}\right|_{(x, u)} \notin N_{\theta}$ for any $\theta \in \mathcal{S}$ and any nonidentity $\mathcal{T} \in G^{\sim}$,

2. $N_{\mathcal{T} \theta}=\left.\mathcal{T}\right|_{(x, u)} N_{\theta}\left(\left.\mathcal{T}\right|_{(x, u)}\right)^{-1}$ for any $\theta \in \mathcal{S}$ and any $\mathcal{T} \in G^{\sim}$,

3. For any $\left(\theta^{1}, \theta^{2}, \varphi\right) \in \mathcal{G}^{\sim}$ there exist $\varphi^{1} \in N_{\theta^{1}}, \varphi^{2} \in N_{\theta^{2}}$ and $\mathcal{T} \in G^{\sim}$ such that $\theta^{2}=\mathcal{T} \theta^{1}$ and $\varphi=\varphi^{2}\left(\left.\mathcal{T}\right|_{(x, u)}\right) \varphi^{1}$,

where $\left.\mathcal{T}\right|_{(x, u)}$ denotes the restriction of $\mathcal{T}$ to the space with local coordinates $(x, u)$. Then the class $\left.\mathcal{L}\right|_{\mathcal{S}}$ is called uniformly semi-normalized with respect to the symmetry-subgroup family $\mathcal{N}_{\mathcal{S}}$.

We end this section by giving the relation between these three properties in terms of point transformations. If we denote by N, USN and SN the set of normalized, uniformly semi-normalized and semi-normalized classes, respectively, then the following relation holds: $N \subset$ USN $\subset$ SN. From this relation we see that each normalized class of differential equations is uniformly semi-normalized and seminormalized, and each uniformly semi-normalized class is semi-normalized. But there are semi-normalized classes that are not uniformly semi-normalized. For instance, the class of nonlinear diffusion equation of the form $u_{t}=\left(f(u) u_{x}\right)_{x}$ with $f \neq 0$ is semi-normalized but not uniformly semi-normalized. 


\section{Group classification problem}

\subsection{Formulation of the problem}

The study of Lie symmetries of differential equations is in general quite complex. It is relatively simple when one investigates an ordinary differential equation since the symmetry analysis can be done by the use of Lie's infinitesimal method. However, this method is not effective when arbitrary elements are involved, and for this situation more advanced techniques are required. We will look at the group classification problem for Schrödinger equations. That is, we will look for the possible symmetries that these equations may possess and classify them according to the types of symmetries that are admitted. The solution of this problem is based on the properties of point transformations that the class under study possesses as well as their equivalence relations.

The group classification problem can be stated as follows: given a class of differential equations $\left.\mathcal{L}\right|_{\mathcal{S}}$ (in an appropriate jet space), find, for a given $\theta \in \mathcal{S}$, all possible inequivalent extensions of the maximal Lie invariance algebra $\mathfrak{g}_{\theta}$ together with all $G^{\sim}$-inequivalent families of the arbitrary element $\theta$ that satisfy the condition $\mathfrak{g}_{\theta} \neq \mathfrak{g}^{\cap}$.

\subsection{Methods to solve the problem of group classification}

Here, we list different cases that may occur when one attempts to solve the group classification problem.

First of all, one finds, for the class $\left.\mathcal{L}\right|_{\mathcal{S}}$, the equivalence groupoid $\mathcal{G}^{\sim}$ and the equivalence group $G^{\sim}$ whose associated equivalence algebra is $\mathfrak{g}^{\sim}$. It is in this step 
that we will know if the class under study possesses the normalization property in view of Section 3.4 .

\subsubsection{Infinitesimal Lie's method and direct integration of determining equations}

For each system $\mathcal{L}_{\theta}, \theta$ fixed from the class $\left.\mathcal{L}\right|_{\mathcal{S}}$, a vector field $X$,

$$
X=\xi^{i}(x, u) \partial_{x_{i}}+\eta^{a}(x, u) \partial_{u^{a}},
$$

belongs to the algebra $\mathfrak{g}_{\theta}$ if and only if it satisfies the infinitesimal Lie invariance criterion, which is the essence of the infinitesimal Lie's method, see Theorem 2.2. This invariance criterion, applied to $X$, leads to the determining equations for the coefficients of the symmetry vector fields in the algebra $\mathfrak{g}_{\theta}$ associated with that equation. This yields an overdetermined systems of linear homogeneous partial differential equations which can then be solved. When the class under study has a simple structure, possesses constant arbitrary elements or a single arbitrary element, the determining equations for Lie symmetries of equations from the class $\left.\mathcal{L}\right|_{\mathcal{S}}$ can be solved by looking at the compatibility analysis and direct integration of these equations (up to equivalence relations). This framework leads to an exhaustive group classification for the class under study.

\subsubsection{Algebraic method for group classification}

For classes with a complex structure, the determining equations lead to overdetermined systems which can be split into two parts: The first which involves arbitrary elements and the second without arbitrary elements. Those which do not involve the arbitrary elements are integrated immediately and give the components of the symmetry vector fields from $\mathfrak{g}_{\theta}$. The remaining equations, those containing arbitrary elements, which we refer to as the classifying equations, constitute the essential part of the group classification of the class $\left.\mathcal{L}\right|_{\mathcal{S}}$. They are investigated in order to obtain Lie symmetry extensions for the kernel invariance algebra $\mathfrak{g}^{\cap}$ (which is obtained by varying the arbitrary elements and further splitting the classifying equations with respect to various powers of the arbitrary element $\theta$ ).

A deeper analysis of the classifying equations depends upon whether the class $\left.\mathcal{L}\right|_{\mathcal{S}}$ is normalized or not. If the class is normalized then the maximal Lie symmetry extensions $\mathfrak{g}_{\theta}$ of the kernel invariance algebra $\mathfrak{g}^{\cap}$ are obtained via the classification of subalgebras of the equivalence algebra, whose projections to the space of independent and dependent variables $(x, u)$ coincide with the maximal Lie invariance algebras of systems from the class $\left.\mathcal{L}\right|_{\mathcal{S}}[24$. This process reduces the group classification problem to the algebraic problem of classifying inequivalent subalgebras of the equivalence algebra $\mathfrak{g}^{\sim}$. In this case, for each system $\mathcal{L}_{\theta}$ with a fixed arbitrary element $\theta$, the solution space of the determining equations is associated with a Lie algebra of vector fields $\mathfrak{g}_{\theta}$, and $\mathfrak{g}_{\theta}$ is contained in the linear span $\mathfrak{g}_{\langle\rangle}:=\sum_{V} \mathfrak{g}_{\theta}$ when $\theta$ varies. The subalgebras of this linear span are called appropriate subalgebras and they contain the kernel invariance algebra $\mathfrak{g}^{\cap}$. This technique for solving the problem of group classification is referred to as the algebraic method. 
If the class under consideration is uniformly semi-normalized with respect to linear superposition of solutions, then it follows from Definition 3.11 that the linear span $\mathfrak{g}_{\langle\rangle}$has a representation of the form

$$
\mathfrak{g}_{\langle\rangle}=\mathfrak{g}_{\langle\rangle}^{\text {ess }} \oplus \mathfrak{g}_{\langle\rangle}^{\operatorname{lin}},
$$

where $\mathfrak{g}_{\langle\rangle}^{\text {ess }}$ and $\mathfrak{g}_{\langle\rangle}^{\text {lin }}$ are a subalgebra and an ideal of $\mathfrak{g}_{\langle\rangle}$, respectively. It follows from this representation that the kernel algebra $\mathfrak{g}^{\cap}$ is an ideal in $\mathfrak{g}_{\langle\rangle}^{\text {ess }}$ and in the whole algebra $\mathfrak{g}_{\langle\rangle}$. The form of the algebra $\mathfrak{g}_{\langle\rangle}$given in such a way induces a similar form of the representation of the maximal Lie invariance algebra $\mathfrak{g}_{\theta}$,

$$
\mathfrak{g}_{\theta}=\mathfrak{g}_{\theta}^{\text {ess }} \Subset \mathfrak{g}_{\theta}^{\operatorname{lin}}
$$

where $\mathfrak{g}_{\theta}^{\text {ess }}:=\mathfrak{g}_{\theta} \cap \mathfrak{g}_{\langle\rangle}^{\text {ess }}$ and $\mathfrak{g}_{\theta}^{\text {lin }}:=\mathfrak{g}_{\theta} \cap \mathfrak{g}_{\langle\rangle}^{\text {lin }}$ are a finite-dimensional subalgebra and an infinite-dimensional abelian ideal of $\mathfrak{g}_{\theta}$, respectively.

Definition 4.1. The algebra $\mathfrak{g}_{\theta}^{\text {ess }}$ with $\mathfrak{g}_{\theta}^{\text {ess }}:=\mathfrak{g}_{\theta} \cap \mathfrak{g}_{\langle\rangle}^{\text {ess }}$ is called the essential invariance algebra corresponding to the system $\mathcal{L}_{\theta}$ of the class $\left.\mathcal{L}\right|_{\mathcal{S}}$.

In this case, the algebra $\mathfrak{g}_{\theta}^{\text {lin }}$ (resp. $\mathfrak{g}_{\langle\rangle}^{\text {lin }}$ ) is considered as the trivial part and the problem of group classification for the class $\left.\mathcal{L}\right|_{\mathcal{S}}$ reduces to the classification of appropriate subalgebras of the algebra $\mathfrak{g}_{\theta}^{\text {ess }}$ up to the equivalence relation generated by the action of $\pi G^{\sim}$.

We close the group classification for normalized classes with the following assertion :

Corollary 4.1. If the class $\left.\mathcal{L}\right|_{\mathcal{S}}$ is normalized in the usual sense then the kernel algebra $\mathfrak{g}^{\cap}$ is an ideal of the maximal Lie algebra $\mathfrak{g}_{\theta}$ for each $\theta \in \mathcal{S}$.

For the proof see [4]. It was shown there that, in general, the kernel invariance algebra $\mathfrak{g}^{\cap}$ is not necessarily an ideal of the maximal Lie invariance algebra $\mathfrak{g}_{\theta}$. This was illustrated by considering the class of $(1+1)$-dimensional non-linear diffusion equation, $u_{t}=\left(F(u) u_{x}\right)_{x}$ with $F \neq 0$, where $\theta=F$. The kernel invariance algebra $\mathfrak{g}^{\cap}$ of this class is $\mathfrak{g}^{\cap}=\left\langle\partial_{t}, \partial_{x}, 2 t \partial_{t}+x \partial_{x}\right\rangle$ and he maximal Lie invariance algebra $\mathfrak{g}_{\theta}$ associated with $F=u^{4 / 3}$ is

$$
\mathfrak{g}_{\theta}=\left\langle\partial_{t}, \partial_{x}, 2 t \partial_{t}+x \partial_{x}, 4 t \partial_{t}+3 u \partial_{u}, \partial_{x}, x^{2} t \partial_{x}-3 x u \partial_{u}\right\rangle
$$

respectively. It is clear seen that $\left[\mathfrak{g}^{\cap}, \mathfrak{g}_{\theta}\right] \not \subset \mathfrak{g}^{\cap}$ since

$$
\left[\partial_{x}, x^{2} t \partial_{x}-3 x u \partial_{u}\right]=2 x \partial_{x}-3 x u \partial_{u} \notin \mathfrak{g}^{\cap} .
$$

In contrast to normalized classes, the group classification of non-normalized classes is done by partitioning the class into disjoint normalized subclasses and then investigating each subclass separately. The solution set of inequivalent Lie symmetry extensions together with their corresponding $G^{\sim}$-inequivalent families of arbitrary elements is the union of all the lists obtained. Both normalized and nonnormalized classes are investigated in our thesis and Lie's infinitesimal method, the algebraic method and direct integrability are used. 


\subsection{Algorithm for solving the problem of group classification}

The solution of the problem of group classification of class of differential equation $\left.\mathcal{L}\right|_{\mathcal{S}}$ requires several steps to reach completion. Here, we indicate the main steps for the complete solution of the group classification problem for Schrödinger equations presented in our thesis.

- Using direct method find the equivalence groupoid $\mathcal{G}^{\sim}$ of the class $\left.\mathcal{L}\right|_{\mathcal{S}}$.

- Construct the equivalence group $G^{\sim}$ of the class $\left.\mathcal{L}\right|_{\mathcal{S}}$ and the equivalence algebra $\mathfrak{g}^{\sim}$ corresponding to this group. This is the main step in the group classification.

- Check the normalization property.

- For an equation $\mathcal{L}_{\theta}$ with a fixed arbitrary element, find Lie symmetries from the determining equations for this equation. Here we derive the maximal Lie invariance algebra $\mathfrak{g}_{\theta}$, the kernel invariance Lie algebra $\mathfrak{g}^{\cap}$ and the classifying equations for Lie symmetry extensions.

- Find all possible $G^{\sim}$-inequivalent values of families of the arbitrary elements $\theta$ together with all inequivalent Lie symmetry extensions of the kernel invariance algebra, (i.e., the algebras $\mathfrak{g}_{\theta}$ such that $\mathfrak{g}_{\theta} \neq \mathfrak{g}^{\cap}$ ).

Summarizing, the problem of group classification is completely solved when the final group classification list contains all the possible inequivalent cases of extensions, all equations from this list are mutually inequivalent with respect to the transformations from the equivalence group $G^{\sim}$ (or the generalized equivalence group $\left.G_{\text {gen }}^{\sim}\right)$ and the Lie symmetry algebras that are obtained are really the maximal invariance algebras of the corresponding equations. 


\section{Summary of the papers}

\section{Paper I: Algebraic method for group classification of $(1+1)$-dimensional linear Schrödinger equations}

We carry out the group classification of the class of $(1+1)$-dimensional linear Schrödinger equations with complex potentials of the form

$$
i \psi_{t}+\psi_{x x}+V(t, x) \psi=0
$$

where $\psi$ is the complex-valued unknown function of the real variables $t$ and $x$, and $V$ is the complex-valued smooth function of $(t, x)$ interpreted as potential.

We find the equivalence groupoid $\mathcal{G}^{\sim}$, the equivalence group $G^{\sim}$ and the equivalence algebra $\mathfrak{g}^{\sim}$ of this class. We show that the class under study is uniformly semi-normalized with respect to linear superposition of solutions. This allows us to apply the algebraic method and reduce the group classification problem of linear Schrödinger equations to the classification of low-dimensional appropriate subalgebras of the equivalence algebra of this class up to the equivalence relations. The dimensional of any maximum Lie symmetry extension of an equation from the above class is proved not to be greater than seven.

Splitting the classification cases with respect to two invariant integers results in eight inequivalent families of potentials together with their corresponding inequivalent Lie symmetry extensions.

\section{Paper II: Group classification of multidimensional linear} Schrödinger equations with the algebraic method

We consider the group classification problem for Schrödinger equations with complex potentials in dimension $(1+n)$ with $n \geqslant 2$,

$$
i \psi_{t}+\Delta \psi+V(t, x) \psi=0,
$$


where $\psi$ is the complex-valued unknown function of the variables $t \in \mathbb{R}$ and $x \in \mathbb{R}^{n}$, and the complex-valued smooth parameter-function $V$ of $(t, x)$ is interpreted as potential.

We find the equivalence groupoid and the equivalence group of the given class in closed form and then show that the class is uniformly semi-normalized. This class admits the similar kernel invariance algebra $\mathfrak{g}^{\cap}$ as the corresponding class investigated in Paper I. We proceed to the calculation of the maximal Lie invariance algebra $\mathfrak{g}_{V}$ of the above Schrödinger equation for each fixed $V$. The algebra $\mathfrak{g}_{V}$ may by essentially higher-dimensional than the algebras obtained in Paper I for the case of $(1+1)$ variables. Moreover, the structure of $\mathfrak{g}_{V}$ may be much more complicated due to appearing rotations in higher-dimensional spaces.

We show that the span $\mathfrak{g}_{\langle\rangle}=\sum_{V} \mathfrak{g}_{V}$ is a Lie algebra and can be represented as the semi-direct sum $\mathfrak{g}_{\langle\rangle}^{\text {ess }} \in \mathfrak{g}_{\langle\rangle}^{\text {lin }}$, where $\mathfrak{g}_{\langle\rangle}^{\text {ess }}$ and $\mathfrak{g}_{\langle\rangle}^{\text {lin }}$ are a subalgebra and an abelian ideal of $\mathfrak{g}_{\langle\rangle}$, respectively.

In order to find Lie symmetry extensions of the kernel invariance algebra $\mathfrak{g}^{\cap}$, we apply the algebraic method and reduce the group classification problem to the classification of certain low-dimensional subalgebras of the associated equivalence algebra. An important role in the consideration is played by estimates of the dimension of the essential Lie invariance algebra $\mathfrak{g}_{V}^{\text {ess }}$ and its various parts for any potential $V$. In particular,

$$
\operatorname{dim} \mathfrak{g}_{V}^{\mathrm{ess}} \leqslant \frac{n(n+3)}{2}+5 .
$$

The group classification of linear Schrödinger equations in the $(1+2)$-dimensional case is considered in more detail. For the exposition to be systematic, we then introduce the three $\pi G^{\sim}$-invariant integers $k_{1} \in\{0,1,2\}, k_{2} \in\{0,1\}$ and $k_{3} \in$ $\{0,1,2,3\}$, respectively. Using these integers, we single out the classification cases and obtain a complete classification of inequivalent Lie symmetry extensions for $(1+2)$-dimensional linear Schrödinger equations with complex-valued potentials.

\section{Paper III: Group classification of multidimensional nonlinear Schrödinger equations}

We study transformational properties for the class $\mathcal{S}$ of multidimensional nonlinear Schrödinger equations,

$$
i \psi_{t}+\Delta \psi+S(t, x, \rho) \psi=0, \quad S_{\rho} \neq 0,
$$

where $t$ and $x=\left(x_{1}, \ldots, x_{n}\right)$ are real independent variables, $\psi$ is the complex dependent variable and $\rho=|\psi|$, and completely carry out the group classification problem of the class $\mathcal{V}$ of $(1+2)$-dimensional Schrödinger equations with potentials and modular nonlinearities of the form $i \psi_{t}+\Delta \psi+f(\rho) \psi+V(t, x) \psi=0$, where $f_{\rho} \neq 0$. We start by computing the equivalence groupoid and equivalence group of the class $\mathcal{S}$ and show that this class is normalized. For a fixed equation from this class we find the Lie symmetry properties of each equation and derive from this the classifying equation as well as the kernel invariance algebra of the class $\mathcal{S}$.

Specifying the form of $S$ as $S=f(\rho)+V(t, x)$ with $f_{\rho} \neq 0$, we obtain the class $\mathcal{V}$, whose the set of admissible transformations does not belong to its equivalence 
group, i.e., the class $\mathcal{V}$ is not normalized, which makes its group classification more involved. To deal with this challenge, we partition the class $\mathcal{V}$ into the three disjoint normalized subclasses $\mathcal{V}^{\prime}, \mathcal{P}_{0}$ and $\mathcal{P}_{\lambda}$, conditioned by $\rho f_{\rho \rho} / f_{\rho} \neq \lambda \forall \lambda \in \mathbb{R}$, $f=\sigma \ln \rho$, and $f=\sigma \rho^{\lambda}$, respectively, where $\sigma \in \mathbb{C} \backslash\{0\}$ and $\lambda \in \mathbb{R} \backslash\{0\}$.

From the knowledge of the equivalence groupoid and the equivalence group of the class $\mathcal{S}$ we derive, successively, the equivalence groupoids and the equivalence groups for the above restricted classes and show that each class is normalized. This allows us to apply the algebraic method for group classification as developed in 4 and reduce the group classification of each subclass to the classification of subalgebras of the associated equivalence algebra. We show that the dimension of the Lie symmetry group of any equation from each class is not greater than $n(n+3) / 2+2, n(n+3) / 2+3$ and $n(n+3) / 2+4$ for $\mathcal{V}^{\prime}, \mathcal{P}_{0}$ and $\mathcal{P}_{\lambda}$, respectively.

The complete group classification of the class $\mathcal{V}$ is carried out for $n=2$. Splitting into different cases, we introduce three integers $k_{1}, k_{2}$ and $k_{3}$ with $\left(k_{1}, k_{2}\right) \in\{0,1\}$ and $k_{3} \in\{0,1,2\}$ for $\mathcal{V}^{\prime}, \mathcal{P}_{0}$ and $k_{1} \in\{0,1,2,3\}, k_{2} \in\{0,1\}$ and $k_{3} \in\{0,1,2\}$ for $\mathcal{P}_{\lambda}$, that characterize the dimension of specific subspaces of the corresponding maximal Lie invariance algebras. As a result, we obtain a complete list of inequivalent Lie symmetry extensions together with the corresponding families of potentials which is made by the union of the three obtained results lists corresponding to each consideration.

\section{Paper IV: Admissible transformations of Schrödinger equations with variable mass and potentials}

We study the admissible transformations in the class $\mathcal{A}$ of $(1+1)$-dimensional generalized nonlinear Schrodinger equations

$$
i \psi_{t}+G\left(t, x, \psi, \psi^{*}, \psi_{x}, \psi_{x}^{*}\right) \psi_{x x}+F\left(t, x, \psi, \psi^{*}, \psi_{x}, \psi_{x}^{*}\right)=0, \quad G \neq 0,
$$

where $t$ and $x$ are independent variables, $\psi$ is the complex dependent variable of $t$ and $x, F$ and $G$ are smooth complex-valued functions of their arguments. Then reduce this study to the class of linear Schrödinger equations with variable mass and complex potentials.

We start by computing the equivalence groupoid of the wide generalized class $\mathcal{H}$ of equations, $i \psi_{t}=H\left(t, x, \psi, \psi^{*}, \psi_{x}, \psi_{x}^{*}, \psi_{x x}, \psi_{x x}^{*}\right)$ with $\left|H_{\psi_{x x}}\right| \neq\left|H_{\psi_{x x}^{*}}\right|$, where $H$ is a complex-valued smooth function of its arguments, which covers the class $\mathcal{A}$, and then find the equivalence groupoid and the equivalence group of the class $\mathcal{A}$. From this, we show that the class $\mathcal{A}$ is not normalized. We partition it with respect to conditions $G=-G^{*}$ and $G \neq-G^{*}$ into two normalized classes, and then investigate the equivalence groupoids and the equivalence groups of the narrowed classes obtained by specifying the forms of the arbitrary functions $G$ and $F$ using the equivalence groupoid of $\mathcal{A}$. We continue this process until we reach the class of $(1+1)$-dimensional linear Schrödinger equations with variable mass and complex potentials singled out by $G=1 / m(t, x)$ and $F=V(t, x) \psi$. Using equivalence transformations of this class, we can gauge the arbitrary element $G$ to the canonical value, i.e., we can set the arbitrary element $G$ to be equal to one. Thus we obtain the class of $(1+1)$-dimensional linear Schrödinger equations with constant mass equal to one and complex potentials, which is well studied in 
Paper 1. Therefore, the group classification of the class of Schrödinger equations with variable mass and complex potentials can be reduced to the group classification of (1+1)-dimensional linear Schrödinger equations with constant mass equal to one and complex potentials.

\section{Paper V: Equivalence groupoid for $(1+2)$-dimensional linear Schrödinger equations with complex potentials}

We compute the equivalence groupoid and the equivalence group of the class of linear Schrödinger equations with complex potentials in dimension $(1+2)$. From the knowledge of these objects we show that this class is semi-normalized. More specifically, any admissible transformations in this class is the composition of a symmetry transformation of the initial equation and an equivalence transformation of the class under study. 


\section{Bibliography}

[1] Bender C.M., Making sense of non-Hermitian Hamiltonians, Rep. Progr. Phys. 70 (2007), 947-1018.

[2] Bender C. M., Brody D. C. and Jones H. F., Complex extension of quantum mechanics, Phys. Rev. Lett., 89 (2002), 4 pp.

[3] Bihlo A., Dos Santos Cardoso-Bihlo E. and Popovych R.O., Enhanced preliminary group classification of a class of generalized diffusion equation, Commun. Nonlinear Sci. Numer. Simul., 16 (2011), 3622-3638.

[4] Bihlo A., Dos Santos Cardoso-Bihlo E. and Popovych R.O., Complete group classification of a class of nonlinear wave equations, J. Math. Phys. 53 (2012), 123515, 32 pp.

[5] Bihlo A. and Popovych R.O., Group classification of linear evolution equations, J. Math. Anal. Appl., 448 (2017), 982-1005.

[6] Bluman G.W. and Kumei S., Symmetries and differential equations, vol. 154, SpringerVerlag, New York, 1989, xiv+412 pp.

[7] Boyer C.P., The maximal 'kinematical' invariance group for an arbitrary potential, Helv. Phys. Acta, 47 (1974), 589-605.

[8] Boyer C. P., Sharp R.T. and Winternitz P., Symmetry breaking interactions for the time dependent Schrödinger equations, J. Math. Phys. 17 (1976), 1439-1451.

[9] Bountis T. and Nobre F. D., Travelling-wave and separated variable solutions of a nonlinear Schrödinger equation, J. Math. Phys., 57(8) (2016).

[10] Doebner H.-D. and Goldin G.A., Properties of nonlinear Schrödinger equations associated with diffeomorphism group representations, J. Phys. A, 27 (1994), 1771-1780.

[11] Doebner H.-D., Goldin G.A. and Nattermann P., Gauge transformations in quantum mechanics and the unification of nonlinear Schrödinger equations, J. Math. Phys., 40 (1999), 49-63.

[12] Fernández F. M., Guardiola R., Ros J. and Znojil, M., A family of complex potentials with real spectrum, J. Phys. A, 32 (1999), 3105-3116.

[13] Fushchich W.I. and Moskaliuk S. S., On some exact solutions of the nonlinear Schrödinger equation in three spatial dimensions, Lett. Nuovo Cimento (2), 31 (1981), 571-576. 
[14] Fushchich W.I., Conditional symmetry of the equations of nonlinear mathematical physics, Ukr. Math. J., 43 (1991), 1350-1364.

[15] Fushchich W.I. and Chopik V.I, Symmetry and non-Lie reduction of the nonlinear Schrödinger equation, Ukr. Math. J., 45 (1993), 581-597.

[16] Fushchych W., Chopyk V., Nattermann P. and Scherer W., Symmetries and reductions of nonlinear Schrödinger equations of doebner-goldin type, Rep. Math. Phys., 35 (1995), $129-138$.

[17] Gagnon L. and Winternitz P., Lie symmetries of a generalised nonlinear Schrödinger equation. II. Exact solutions, J. Phys. A, 22 (1989), 469-497.

[18] Gagnon L., Grammaticos B., Ramani A. and Winternitz P., Lie symmetries of a generalised nonlinear Schrödinger equation. III. Reductions to third-order ordinary differential equations, J. Phys. A, 22 (1989), 499-509.

[19] Gagnon L. and Winternitz P., Exact solutions of the cubic and quintic nonlinear Schrödinger equation for a cylindrical geometry, Phys. Rev. A (3), 39 (1989), 296-306.

[20] Gagnon L. and Wintenitz P., Symmetry classes of variable coefficient nonlinear Schrödinger equations, J. Phys. A, 26 (1993), 7061-7076.

[21] Griffin A., Snoke D. W. and Stringari S. Bose-Einstein Condensation. Cambridge University Press, 1996.

[22] Ivanova N.M, Popovych R.O. and Sophocleous C., Group analysis of variable coefficient diffusion-convection equations. I. Enhanced group classification, Lobachevskii Journal of mathematics, 31(2) (2010), 100-122.

[23] Kurujyibwami C., Equivalence groupoid for (1+2)-dimensional linear Schrödinger equations with complex potentials, J. Phys.: Conf. Ser., 621 (2015), 12008-12014.

[24] Kurujyibwami C., Basarab-Horwath P. and Popovych R.O., Algebraic method for group classification of (1+1)-dimensional linear Schrödinger equations, (Submitted to Acta Appl. Math.) (2016), arXiv:1607.04118, 30 pp.

[25] Miller W., Symmetry and separation of variables, Addison-Wesley Publishing Co., Reading, MA, 1977.

[26] Mostafazadeh A., A dynamical formulation of one-dimensional scattering theory and its applications in optics, Ann. Physics, 341 (2014), 77-85.

[27] Niederer U., The maximal kinematical invariance group of the free Schrödinger equation, Helv. Phys. Acta, 45 (1972), 802-810.

[28] Niederer U., The group theoretical equivalence of the free particle, the harmonic oscillator and the free fall, Helv. Phys. Acta, (1973).

[29] Niederer U., The maximal kinematical invariance groups of the harmonic oscillator, Helv. Phys. Acta., 46 (1973), 191-200.

[30] Niederer U., The maximal kinematical invariance groups of Schrödinger equations with arbitrary potentials, Helv. Phys. Acta 47 (1974), 167-172.

[31] Nikitin A.G. and Popovych R.O, Group classification of nonlinear Schrödinger equations, 47 (2003), arXiv preprint math-ph/0301009, 11pp.

[32] Noether E., Invariante variationsprobleme, nachr. d. könig. gesellsch. d. wiss. zu göttingen, math-phys. klasse (1918), 235-257; english translation ma travel, Transp. Theory. Stat. Phys., 1 (1971), 183-207.

[33] Olver P.J., Applications of Lie groups to differential equations, Springer-Verlag, New York, 1993.

[34] Ovsiannikov L.V., Group properties of nonlinear heat equation, dokl, AN SSSR, 125 (1959), $492-495$

[35] Ovsiannikov L.V., Group properties of differential equations, (Izdat. Sibirsk. Otdel. Akad. Nauk SSSR, Novosibirsk) Siberian Section of the Academy of Science of USSR, 1962, 239 pp. 
[36] Ovsyannikov L.V., and Ibragimov N.K., Group analysis of the differential equations of mechanics, 2, 1975.

[37] Ovsiannikov L.V., Group analysis of differential equations, Academic Press, New-York, 1982, xvi+416 pp.

[38] Pommaret J.F., Systems of partial differential equations and Lie pseudogroups, Gordon and Breach Science Publishers, New York, 1978, xiv+411 pp.

[39] Popovych R.O, Normalized classes of nonlinear Schrödinger equations, Bulg. J. Phys, 33 (2006), 211-222.

[40] Popovych R.O., Kunzinger M. and Eshraghi H., Admissible transformations and normalized classes of nonlinear Schrödinger equations, Acta Appl.Math., 109 (2010), 315-359, arXiv:math-ph/o611061.

[41] Saunders D.J., The geometry of jet bundles, Cambridge University Press, Cambridge University Press, Cambridge, 1989, viii+293 pp.

[42] Srivastava V.K. and Bose S.K, Exact solution of relativistic Schrödinger equation for the central complex potential $V(r)=i a r+(b / r)$, Indian J. Pure Appl. Phys., 47 (2009), $547-550$.

[43] Torres P.J., Lie Symmetries, qualitative analysis and exact solutions of nonlinear Schrödinger equations with inhomogeneous nonlinearities, Discrete Continuous. Dyn. Syst. Ser B., 9, (2007) 221-233.

[44] Vaneeva O. and Posta S., Equivalence groupoid of a class of variable coefficient Korteweg-de Vries equations, (2016), arXiv preprint arXiv:1604.06880, 15 pp.

[45] Varadarajan V., Lie groups, Lie algebras, and their representations, Springer Science and Business Media, 2013.

[46] Zhdanov R. and Roman O., On preliminary symmetry classification of nonlinear Schrödinger equations with some applications to Doebner-Goldin models, Rep. Math. Phys., 45 (2000), 273-291. 


\section{Papers}

The articles associated with this thesis have been removed for copyright reasons. For more details about these see:

http://urn.kb.se/resolve? urn:nbn:se:liu:diva-137424 